\title{
Using Cluster Abundances and Peculiar Velocities to Test the Gaussianity of the Cosmological Density Field
}

\author{
Weihsueh A. Chiu \\ Joseph Henry Laboratories, Department of Physics, Princeton, NJ 08544 \\ Jeremiah P. Ostriker and Michael A. Strauss' \\ Princeton University Observatory, Princeton, NJ 08544
}

\begin{abstract}
Comparing the frequency of typical events with that of unusual events allows one to test whether the cosmological density distribution function is consistent with the normally made assumption of gaussianity. To this end, we compare the consistency of the tail-inferred (from clusters) and measured values (from largescale flows) of the rms level of mass fluctuations for two distribution functions: a Gaussian, and a texture (positively-skewed) PDF. We find that if we average the recent large-scale flow measurements, observations of the rms and the tail at the $10 h^{-1} \mathrm{Mpc}$ scale disfavor a texture PDF at $\sim 1.5 \sigma$ in all cases. If we take the most recent measurement of the rms, that from Willick et al. (1997b), the comparison disfavors textures for low $\Omega_{0}=0.3$, and disfavors Gaussian models if $\Omega_{0}=1$ (again at $\sim 1.5 \sigma$ ). Predictions for evolution of high temperature clusters can also be made for the models considered, and, as is known (e.g., Henry 1997), strongly disfavor $\Omega_{0}=1$ Gaussian models, while we find $\Omega_{0}=1$ marginally disfavored in texture models. Taking the suite of tests as a whole, and using all of the quoted data, it appears that textures are strongly disfavored and only the low $\Omega_{0}$ Gaussian models are consistent with all the data considered. But given evidence for the internal inconsistency of the observational data, had we only used the recent Willick et al. results, the strength of our conclusion would have been reduced to the $\sim 1 \sigma$ level.
\end{abstract}

Subject headings: cosmology: large-scale structure of universe — galaxies: clusters and statistics — methods: analytical

\footnotetext{
${ }^{1}$ Alfred P. Sloan Foundation Fellow, and Cottrell Scholar of Research Corporation.
} 


\section{Introduction}

All current scenarios for the formation of structure in the Universe assume that cosmic structures grow from initially small seed perturbations. A fundamental assumption underlying a large class of these theories is that the initial Fourier modes have random phases. This implies that the statistics of the initial density field $\rho_{i}(\mathbf{x})$ are fully specified by the correlation function $\xi_{i}(r)=\left\langle\rho_{i}(\mathbf{x}) \rho_{i}(\mathbf{x}+\mathbf{r})\right\rangle_{\mathbf{x}}$, or equivalently its Fourier transform, the power spectrum $P_{i}(k)$. For any given smoothing length, then, the one-point probability distribution function (PDF) of the initial density field $\rho_{i}$ is Gaussian. A measurement of the frequency of rare events (clusters of galaxies) coupled with a measurement of the level of rms fluctuations can tell us directly whether or not the distribution of density fluctuations satisfy the Gaussian hypothesis.

While theories based on the inflationary paradigm generally predict Gaussian initial conditions, there are many alternatives which do not. Global textures and other topological defects, for example, predict a strongly non-Gaussian distribution for the initial perturbations (e.g., Gooding, Spergel, \& Turok 1991; Park, Spergel, \& Turok 1991; Gooding et al. 1992; Pen, Spergel, \& Turok 1994; Pen et al. 1997). Global textures arise when a global non-Abelian symmetry, such as $\mathrm{SU}(2)$, is spontaneously and completely broken. When these defects collapse, energy gradients in the Higgs field perturb the metric and induce gravitational fluctuations in the matter fields. These texture knots, then, act as "causal seeds" of structure formation. We take the texture model as our standard non-Gaussian model, since it is well studied and still viable as a theory of structure formation. In general, texture and Gaussian models give similar predictions for the shape of the power spectrum and the level of CMB fluctuations (Pen, Spergel, \& Turok 1994; Pen \& Spergel 1995). In fact, it is often noted (e.g., Park, Spergel, \& Turok 1991) that texture and Gaussian CDM models are most different in their PDFs. If it could be demonstrated that the initial density field were Gaussian on some suitable scale, the texture model would be directly falsified.

The standard method for testing Gaussianity relies on measuring the $N$-th order reduced moments, or cumulants $\lambda_{N}$ of the present-day PDF, such as skewness or kurtosis, from redshift or peculiar velocity surveys (cf., Strauss \& Willick 1995 for a review). The variance is $\sigma^{2} \equiv \lambda_{2} \equiv\left\langle\delta^{2}\right\rangle$, the skewness is $\lambda_{3} \equiv\left\langle\delta^{3}\right\rangle$, and the kurtosis is $\lambda_{4} \equiv\left\langle\delta^{4}\right\rangle-3 \sigma^{4}$, where $\delta$ is the density contrast $\rho /\langle\rho\rangle-1$. For a Gaussian random field, all the cumulants of order $N>2$ are equal to zero. The growth of Gaussian initial perturbations under gravitational instability naturally produces non-Gaussianity in the distribution function of the density field. In particular, the cumulants follow a scaling relation $\lambda_{N}=S_{N} \lambda_{2}^{N-1}$, where the $S_{N}$ are independent of scale in the mildly non-linear regime for power-law power spectra (Fry 1984ab, Bernardeau 1992), and can be calculated from $(N-1)$-th order perturbation theory 
(Bernardeau 1994). Significant departures from scale-invariance are taken as signs of nonGaussianity. Unfortunately, the higher-order moments are very sensitive to finite-volume effects and non-Gaussian noise in the data (c.f. the discussion in Kim \& Strauss 1997 and references therein).

Since many non-Gaussian distribution functions have long tails, it is better to directly compare the rms and the tail of the PDF, rather than relying on the moments, which are integrals over the PDF. Given a distribution function, the level of tail fluctuations maps directly to a level of rms fluctuations; the tail-inferred rms, then, must be consistent with the directly measured rms. Furthermore, one should look for measures which are, as closely as possible, indicators of the initial, linear density field.

In this paper, we use the observed temperature distribution of clusters of galaxies as a measure of the tail of the distribution of initial density fluctuations, and galaxy peculiar velocities and redshift-space distortions as measures of the rms. Clusters of galaxies are the most massive virialized objects in the universe, with densities a hundred or more times that of the mean density of the universe, containing only a few percent of all galaxies. They are thus extreme excursions from the mean, and hence are measures of the high potential tail of the distribution of fluctuations. We compare this with the rms level of peculiar velocity fluctuations. The rms is not as sensitive to non-linear growth as are higher order statistics (Dekel, private communication). Both cluster and peculiar velocity observations are at roughly the same spatial scale, $\sim 10 h^{-1} \mathrm{Mpc}$. Furthermore, they both measure gravitational potential fluctuations, and therefore have a similar dependence on $\Omega_{0}$, which can be approximated by defining

$$
\eta_{R} \equiv \sigma_{R} \Omega_{0}^{0.6}
$$

where $\sigma_{R}$ is the rms mass fluctuation on tophat filter scale $R$. The remaining $\Omega_{0}$ dependence is $\leq 5 \%$ for the peculiar velocity and redshift distortion measurements, and $\leq 25 \%$ in the range $0.3 \leq \Omega_{0} \leq 1.0$ for the cluster measurements. This is the quantity we will attempt to determine throughout our analysis, comparing estimates of it found by various methods.

In $\S 2$, we examine various recent derivations of $\eta_{10}$ from the Mark III peculiar velocity catalog (Willick et al. 1995, 1996, 1997a), and from IRAS galaxy redshift space distortions. In $\S 3$, we infer $\eta_{10}$ from the redshift-zero X-ray cluster abundances, assuming a Gaussian and texture PDF, using a modified Press-Schechter approach. We calculate the $\eta_{10}$ separately for $\Omega_{0}=1.0$, cosmologically flat $\left(\Lambda\right.$-dominated) $\Omega_{0}=0.3$, and open $\Omega_{0}=0.3$. In $\S 4$, we discuss the consistency of the rms and the tail-inferred measures of $\eta$ by performing a likelihood analysis of the Gaussian and texture hypotheses. We also discuss further constraints from observations of clusters at moderate redshift. We summarize in $\S 5$. 


\section{RMS Mass Fluctuations from Peculiar Velocities and Redshift Distortions}

It has been noted for more than a decade that the rms optical galaxy counts in $8 h^{-1} \mathrm{Mpc}$

spheres is $\left\langle(\delta N / N)_{8}^{2}\right\rangle^{1 / 2} \equiv \sigma_{8}^{\text {Optical }} \approx 1$ (Davis \& Peebles 1983). But given that galaxy formation is still not very well understood, it has become customary to relate this measured variance to the more fundamental rms fluctuations in the matter density $\sigma_{8}$ by a bias parameter $b$. For optical galaxies, $b^{\text {Optical }}$ is defined by $\sigma_{8}^{\text {Optical }} \equiv b^{\text {Optical }} \sigma_{8}$. The bias, however, is clearly dependent on the galaxy type (for example, as we will see below $\sigma_{8}^{I R A S}=0.69 \pm 0.04$, Fisher et al. 1994a), and may be dependent on scale. We wish to estimate the rms value of fluctuations independent of bias. To do this, we look at recent analyses of peculiar velocity and redshift survey data.

\subsection{Recent Measurements of RMS Fluctuations}

The peculiar velocity $\mathbf{v}$ is the deviation of the proper velocity of a galaxy from the local Hubble flow. Assuming that this measured velocity follows from gravitational potential fluctuations, we obtain from linear perturbation theory (Peebles 1993):

$$
\nabla \cdot \mathbf{v}=-H_{\circ} f\left(\Omega_{0}\right) \delta \approx-H_{\circ} \Omega_{0}^{0.6} \delta .
$$

Here $\delta$ is the mass density contrast $\delta(\mathbf{x}) \equiv \rho_{\operatorname{lin}}(\mathbf{x}) / \bar{\rho}-1$, and the approximation is good to $5 \%$. Now consider filtering the velocity and density fields with a tophat of radius $R$, yielding the filtered fields $\mathbf{v}_{R}$ and $\delta_{R}$. Then $\nabla \cdot \mathbf{v}_{R}$, the divergence of the filtered velocity field, is proportional to the filtered mass density field $\delta_{R}$. Taking the rms values of $\nabla \cdot \mathbf{v}_{R}$ and $\delta_{R}$ gives:

$$
H_{\circ}^{-1}\left\langle\left(\nabla \cdot \mathbf{v}_{R}\right)^{2}\right\rangle^{1 / 2} \approx \Omega_{0}^{0.6}\left\langle\delta_{R}^{2}\right\rangle^{1 / 2} \equiv \Omega_{0}^{0.6} \sigma_{R} \equiv \eta_{R} .
$$

The peculiar velocity field thus provides a direct measurement of mass fluctuations, appearing with the cosmological density parameter $\Omega_{0}$. Note that in linear theory, where individual Fourier components evolve independently, it makes no difference in which order one filters and takes the divergence of the velocity field.

Seljak \& Bertschinger (1993) made an early attempt to measure $\eta_{R}$ directly using the POTENT method (Bertschinger et al. 1990) to reconstruct the mass density and peculiar velocity fields from the Mark II catalog (Burstein 1989). They derived a value of $\eta_{8}=$ $1.3 \pm 0.3$. More recently, several workers have used the Mark III catalog of peculiar velocities (Willick et al. 1995, 1996, 1997a) to derive values of $\eta$.

Zaroubi et al. (1997) perform a likelihood analysis of the peculiar velocities given families of CDM-type models. Because we are making a comparison only with clusters of galaxies, 
which are measures of fluctuations on scales of roughly $10 h^{-1} \mathrm{Mpc}$, we only consider their COBE-independent results. Zaroubi et al. (1997) work with the measured peculiar velocities of individual galaxies, without any smoothing, so it is unclear to which scale they are most sensitive. We simply integrate their power spectra with $10 h^{-1} \mathrm{Mpc}$ tophat smoothing to obtain the rms fluctuation on that scale. Their "maximum likelihood" model has $\eta_{10}=0.70$, and the models on their " $65 \%$-confidence" level contour span the range $\eta_{10}=0.61-0.78$. We therefore take their measurement to be $\eta_{10}=0.70 \pm 0.09$.

Kolatt \& Dekel (1997) use the POTENT technique (Dekel et al. 1990) to determine the quantity $\nabla \cdot \mathbf{v}_{R}$ from the Mark III peculiar velocities, to compute the mass power spectrum directly, subtracting out a model for the noise power spectrum derived from mock catalogs. Since POTENT applies a Gaussian filter of filter length $12 h^{-1} \mathrm{Mpc}$, equivalent to a $\sim 23 h^{-1} \mathrm{Mpc}$ tophat filter (see Appendix), we integrate the functional form they give for the observed power spectrum, taking into account the uncertainties in the parameterization, to derive a value of $\eta_{23}=0.32 \pm 0.11$.

If we assume linear biasing, $\delta_{\text {galaxies }}=b \delta_{\text {dark matter, }}$, comparison of peculiar velocity and galaxy density field data via equation (2) allows one to determine the quantity $\beta \equiv \Omega_{0}^{0.6} / b$, the proportionality constant between the peculiar velocity and galaxy density fields for a particular set of galaxies. Note that if bias is independent of scale, $\beta$ should be as well. Following equation (3) and the definition of $b$, we find that for a comparison with IRAS galaxies:

$$
\eta_{R}=\beta_{I R A S} \sigma_{R, I R A S}
$$

For $\sigma_{R, I R A S}$, we use the power law analytic approximation of Fisher et al. (1994a):

$$
\sigma_{R}=\left[\frac{72\left(r_{\circ} / R\right)^{\gamma}}{2^{\gamma}(3-\gamma)(4-\gamma)(6-\gamma)}\right]^{1 / 2} ; \quad r_{\circ}=3.76_{-0.23}^{+0.20} h^{-1} \mathrm{Mpc} ; \quad \gamma=1.66_{-0.09}^{+0.12}
$$

Several groups have determined $\beta$ from comparisons of IRAS density and peculiar velocity fields. Willick et al. (1997b) perform a likelihood analysis of Tully-Fisher observables of the Mark III data given a prior velocity model, and find $\beta^{I R A S}=0.49 \pm 0.07$. Their analysis uses an effective Gaussian smoothing of $\sim 4 h^{-1} \mathrm{Mpc}$ and so corresponds roughly to an $8 h^{-1} \mathrm{Mpc}$ tophat. Using $\sigma_{8}^{I R A S}=0.69 \pm 0.04$, we obtain $\eta_{8}=0.34 \pm 0.05$. In another approach, Sigad et al. (1997) compare the POTENT-derived peculiar velocity divergence field to the IRAS galaxy density field (with effective tophat smoothing of $\sim 23 h^{-1} \mathrm{Mpc}$ due to the POTENT method) and derive $\beta=0.89 \pm 0.12$. Multiplying by $\sigma_{23}^{\text {IRAS }}=0.29 \pm 0.02$ leads to $\eta_{23}=0.26 \pm 0.04$.

Finally, as first pointed out by Kaiser (1987), one can use the anisotropy of the redshiftspace correlation function of a redshift survey to infer the value of $\beta$. Fisher et al. (1994b) 
measured the anisotropy of the IRAS 1.2Jy sample at an effective scale of $10-15 h^{-1} \mathrm{Mpc}$, obtaining $\beta=0.45_{-0.18}^{+0.27}$. Using the same procedure as above to convert $\beta$ to $\eta$, we obtain $\eta_{12.5}=0.21 \pm 0.11$. Cole et al. $(1994,1995)$ use instead the power spectrum anisotropy, at an effective scale of $\sim 35 h^{-1} \mathrm{Mpc}$, to obtain $\beta=0.52 \pm 0.13$, resulting in $\eta_{35}=0.11 \pm$ 0.03. Yet another method involves decomposing the redshift-space distribution into spherical harmonics. Fisher, Scharf, \& Lahav (1994) (c.f. Heavens \& Taylor 1995) show that the inferred value of $\beta$ depends sensitively on the assumed power spectrum. Scaling their results for each of the power spectra they use, we find that the results for $\eta$ roughly converge at $30 h^{-1} \mathrm{Mpc}$, with a value $\eta_{30}=0.20 \pm 0.03$.

Table 1 summarizes the values of $\eta_{R}$ cited above, all using rms fluctuations, while Figure 1 plots all the recent measurements, with their respective errors, at their effective tophat filter scales. Most current theories of structure formation predict that $\sigma_{R}$ is approximately a power law in the range $8<R<35 h^{-1} \mathrm{Mpc}$. We thus approximate $\eta_{R}$ as a power law $\eta_{R} \propto R^{-\alpha}$, to allow us to extrapolate the results of Table 1 1 and Figure 1 to a common scale. In linear theory, a standard CDM model with $n=1$ and $\Omega_{0}=1$ has a logarithmic slope at $10 h^{-1} \mathrm{Mpc}$ of $\alpha \approx 1.0$, while a tilted $n=0.7$, low density $\Omega_{0}=0.3$ CDM model has $\alpha \approx 0.6$. An $\Omega_{0}=1$ texture model (using the power spectra of Pen, Spergel, \& Turok 1994 and Pen $\&$ Spergel 1995) gives $\alpha \approx 1.2$ at $10 h^{-1} \mathrm{Mpc}$. If galaxy bias does not change appreciably in this range, then $\alpha$ is the same as that for galaxies. Equation (5) implies an exponent $\alpha=0.83 \pm 0.12$ for galaxy fluctuations. If the true value of $\alpha$ for mass fluctuations is much less (greater) than 0.83 , then bias must decrease (increase) with increasing scale. When extrapolating the above results to different scales, then, we take three different power laws to reflect this uncertainty in the slope of the fluctuation spectrum, $\eta_{R} \propto R^{-\alpha}: \alpha=0.83,0.60$, and 1.10. Table 1 contains extrapolations of $\eta_{R}$ to $10 h^{-1} \mathrm{Mpc}$ for each of these assumed values for $\alpha$. It is striking that the different methods give answers which differ from one another by significantly more than the quoted errors - a sure sign that systematic errors of unknown origin are important. We now present a method for combining these measurements.

\subsection{An Error Model for the Different Measurements of $\eta$}

Let us assume that each of the seven measurements (labeled $i=1 \ldots 7$ ) listed in Table 1 has an unknown systematic error $\mu_{i}$ from the true value of $\eta$. That is, each one actually measures $\eta+\mu_{i}$, rather than $\eta$, where the stated statistical error $\epsilon_{i}$ is the error in the measurement of $\eta+\mu_{i}$. We assume that the systematic error is drawn from a Gaussian distribution with zero mean and unknown variance $\theta^{2}$, and that the statistical errors $\epsilon_{i}$ are also Gaussian distributed. The probability of a measurement $x_{i}$, given unknown $\eta$ and $\theta$, is 
given by

$$
\begin{aligned}
\mathcal{P}\left(x_{i} \mid \eta, \theta\right) & \equiv \int d \mu_{i} \mathcal{P}\left(x_{i} \mid \mu_{i}, \eta\right) \mathcal{P}\left(\mu_{i} \mid \theta\right) \\
& =\frac{1}{\sqrt{2 \pi\left(\theta^{2}+\epsilon_{i}^{2}\right)}} \exp \left[-\frac{\left(x_{i}-\eta\right)^{2}}{2\left(\theta^{2}+\epsilon_{i}^{2}\right)}\right] .
\end{aligned}
$$

The systematic error simply adds an error $\theta$ in quadrature to each measurement error $\epsilon_{i}$.

If we define the likelihood function of the unknowns $\eta$ and $\theta$ in the usual way, we obtain

$$
\begin{aligned}
\ln \mathcal{L}(\eta, \theta) & \equiv \ln \left[\prod_{i} \mathcal{P}\left(x_{i} \mid \eta, \theta\right)\right] \\
& =-\frac{1}{2} \sum_{i}\left[\frac{\left(x_{i}-\eta\right)^{2}}{\theta^{2}+\epsilon_{i}^{2}}+\ln \left[2 \pi\left(\theta^{2}+\epsilon_{i}^{2}\right)\right]\right] .
\end{aligned}
$$

The maximum likelihood value for $\eta$ is obtained by setting the derivative of equation (9) to zero:

$$
\hat{\eta}=\left(\sum_{i} \frac{x_{i}}{\theta^{2}+\epsilon_{i}^{2}}\right) \times\left(\sum_{i} \frac{1}{\theta^{2}+\epsilon_{i}^{2}}\right)^{-1} .
$$

Note that equation (10) gives the traditional weighted average for $\theta \ll \epsilon_{i}$, and an unweighted average for $\theta \gg \epsilon_{i}$. Since we are primarily concerned with $\eta$, we numerically integrate the likelihood $\mathcal{L}(\eta, \theta)$ over $\theta$ to obtain the marginal likelihood over $\eta$ :

$$
\mathcal{L}_{\text {rms }}(\eta)=\int_{0}^{\infty} \mathcal{L}(\eta, \theta) d \theta
$$

In Figure \&, we show the likelihood $\mathcal{L}_{\text {rms }}(\eta)$, each normalized to $\int d \eta \mathcal{L}=1$, in the case of $\alpha=0.60,0.83$, and 1.10 , along with a Gaussian distribution of the same $68 \%$ confidence level in the case of $\alpha=0.83$. Note the significant tail to the distribution relative to a Gaussian, reflecting the fact that the measurements are not consistent their individual normal errors. The values of $\eta_{10}$ corresponding to the maximum of $\mathcal{L}_{\text {rms }}(\eta)$, equation (11), along with $1 \sigma$ (68\% confidence) errors, are given in the last row of Table 1, for various $\alpha$. For $\alpha=0.83$, the rms measurements of the velocity field give $\eta_{10}=0.44 \pm 0.08(1 \sigma)$.

This model is only a rough attempt at quantifying the degree of systematic errors present in these measurement. The assumptions that each systematic error $\mu_{i}$ is independent and normally distributed are certainly not completely correct. All of the analyses use the IRAS galaxy redshift catalog, and many of them use the same Mark III catalog, and hence their results must be somewhat correlated. Furthermore, systematic errors are rarely normally distributed. Perhaps one of the measurements is correct, and all the others are erroneous. In comparing rms and tail-inferred values of $\eta$, we therefore also use the Willick et al. (1997b) result alone, which is the most recent result which explicitly filters at a scale close to the $\sim 10 h^{-1} \mathrm{Mpc}$ scale from which clusters form. 


\section{Tail Fluctuations: Abundances of X-ray Clusters}

Galaxy statistics and peculiar velocities measure the rms fluctuations of the density field - i.e., fluctuations within $\sim 1 \sigma$ from the mean. Most probability distributions look similar to Gaussians at this fluctuation level - they are peaked and convex near the mean. The more dramatic differences between Gaussian and non-Gaussian distributions come in their tails. Rich clusters of galaxies, the most massive virialized objects in the universe, offer the best measure of the tail of the probability distribution of the density field on scales of $\sim 10 h^{-1} \mathrm{Mpc}$. Approximately $5 \%$ of the $L_{*}$ galaxies are within one Abell radius of a rich cluster (Bahcall 1996), and about $10 \%$ of the baryons reside in clusters (taking a BBN value of $\Omega_{b}=0.0125 h^{-2}$ from Walker et al. 1991). Thus in the Gaussian scenario, they constitute $\gtrsim 1.5 \sigma$ perturbations. They are well-observed by a number of techniques; in particular, their abundance as a function of X-ray temperature is well-measured (Henry \& Arnaud 1991). Thus they provide an accurate estimate of the integral over the tail of the distribution function at the $\gtrsim 1.5 \sigma$ level.

The temperature function of rich clusters can be predicted from the statistics of peaks in a density field; it depends on the rms level of fluctuations $\sigma_{R}$ on the scale on which clusters form, as well as $\Omega_{0}$ through the relation between comoving radius and mass. Comparing these predictions with observations constrains the quantity $\sigma_{R} \Omega_{0}^{\nu}, \nu=0.4 \sim 0.6$ (cf., White, Efstathiou, \& Frenk 1993; Eke, Cole, \& Frenk 1996; Pen 1997, and references therein). The mass of a rich cluster within the $1.5 h^{-1} \mathrm{Mpc}$ Abell radius is $10^{14}-2 \times 10^{15} h^{-1} M_{\odot}$, corresponding to a region with initial comoving radius $R=\left[3 M /\left(4 \pi \bar{\rho}_{\circ}\right)\right]^{1 / 3}=(4.4-12) \Omega_{0}^{-1 / 3} h^{-1} \mathrm{Mpc}$. Numerical simulations have confirmed that the cosmic abundance of these objects is determined by the power spectrum at $k^{-1}=5-20 h^{-1} \mathrm{Mpc}$, so that their frequency provides a direct measure of $\sigma_{R}$ in this range (e.g., Bahcall \& Cen 1992; Evrard et al. 1996; Cen 1997, in preparation). Hence the cluster abundances constrain $\eta_{10}$ with only a weak dependence on $\Omega_{0}$. In $\S 3.1$ we derive the relation between the distribution of clusters as a function of temperature and the assumed density PDF. This requires a relation between the observed temperature of a cluster and the initial comoving radius from which it formed; this is given in $§ 3.2$. This relation depends on the formation epoch of the cluster, as derived in $\S 3.3$. We compare these results with the observed temperature distribution of clusters in $\S 3.4$ to derive the quantity $\eta_{10}$. 


\subsection{Press-Schechter and the Temperature Function}

We use the Press-Schechter (1974) ansatz (hereafter PS) to derive the number density of clusters. Many have done this in the Gaussian case (e.g., White et al. 1993), and here we make a straight-forward generalization to non-Gaussian distributions. In PS, an object of mass $\geq M$ forms when the linearly-extrapolated density contrast filtered on a mass scale $M$, corresponding to a tophat comoving length scale $R$, exceeds the threshold $\delta_{c} \approx 1.69$. The exact value of $\delta_{c}$ depends on $\Omega_{0}$ and $\Lambda$, and can be derived assuming uniform spherical collapse (see Eke, Cole, \& Frenk 1996), but varies by only a few percent for $\Omega_{0}>0.2$. The differential abundance from PS, given here as the comoving number density at the present of objects with initial comoving radius in the interval $(R, R+d R)$, can be written as

$$
n_{R} d R=\frac{3}{4 \pi R^{3}}\left|\frac{d}{d R} \int_{\delta_{c} / \sigma_{R}}^{\infty} f P(y) d y\right| d R,
$$

where we assume that the mass distribution function $P(y)$ only depends on $y \equiv \delta / \sigma_{R}$. This is true in Gaussian theories; in the texture model, the seeding of density perturbations is scaleinvariant, and thus the distribution function must also be scale-invariant. The prefactor $f \equiv\left[\int_{0}^{\infty} P(y) d y\right]^{-1}$ normalizes $P$ so that all the mass in the universe is accounted for. Assuming, as before, a power law dependence $\sigma_{R} \propto R^{-\alpha}$ gives

$$
n_{R}=\frac{3}{4 \pi R^{3}} \frac{\alpha y}{R} f P(y), \quad y \equiv \frac{\delta_{c}}{\sigma_{R}} .
$$

The initial comoving radius is of course not an observable, so we will convert to the abundance as a function of present-day temperature:

$$
n_{T} \frac{d T}{d R}=\frac{3}{4 \pi R^{3}} \frac{\alpha y}{R} f P(y) .
$$

The observed X-ray temperature function gives the $n_{T}$ of equation (14) as a function of temperature. In order to find $y$ (and hence $\sigma$ and $\eta$ ), we need the appropriate PDF for each model, and the relationship between the initial comoving radius and the temperature of the clusters (next section).

In Gaussian theories, the distribution function is

$$
f P(y)=\frac{2}{\sqrt{2 \pi}} \exp \left(-y^{2} / 2\right)
$$

recalling that the factor $f \equiv\left[\int_{0}^{\infty} P(y) d y\right]^{-1}=2$. For textures, we take the distribution function from the numerical simulations of Park, Spergel, \& Turok (1991), which are the 
only published simulations which are at scales appropriate for clusters of galaxies. We find that the high- $y$ tail of their distribution gives:

$$
f P(y)=2.2 \exp (-1.45 y), \quad y \geq 1.5 .
$$

Equation (16) is not valid for $y<1.5$, but as we are only considering the tail of the distribution, it suffices for our purposes. We show the tails of the functions $f P(y)$ for a Gaussian and texture PDF in Figure 3. Note that the texture PDF has a significantly wider tail for $y \gtrsim 2$.

\subsection{The Radius-Temperature Relation}

We next consider the relationship between the initial comoving radius of the perturba-

tion and the present temperature of a cluster. We make the standard assumption that a cluster forms by undergoing a spherical collapse to a singular isothermal sphere with a virial radius equal to half its maximum expansion radius (e.g., White et al. 1993, Eke et al. 1996, Pen 1997). The X-ray temperature simply reflects the depth of the gravitational potential well of the cluster. We assume a hydrogen mass fraction of 0.76 . For clusters collapsing at redshift $z_{f}$, we obtain

$$
\begin{aligned}
T & =\frac{\mu m_{p} G M}{2 r_{\mathrm{vir}}} \\
& =7.8 \mathrm{keV}\left(\frac{M}{10^{15} h^{-1} M_{\odot}}\right)^{2 / 3}\left(\frac{\Delta_{c}\left(z_{f}\right)}{18 \pi^{2}}\right)^{1 / 3}\left(\frac{\Omega_{0}}{\left.\Omega_{(}\right)}\right)^{1 / 3}\left(1+z_{f}\right) \\
& =8.6 \mathrm{keV} \Omega_{0}^{2 / 3}\left(\frac{R}{10 h^{-1} \mathrm{Mpc}}\right)^{2}\left(\frac{\Delta_{c}(0)}{18 \pi^{2}}\right)^{1 / 3} \times\left[\left(\frac{\Delta_{c}\left(z_{f}\right)}{\Delta_{c}(0)} \cdot \frac{\Omega_{0}}{\Omega\left(z_{f}\right)}\right)^{1 / 3}\left(1+z_{f}\right)\right] \\
& \equiv T_{0}(R) \times \phi\left(z_{f}\right)
\end{aligned}
$$

where the quantity in the square brackets is designated $\phi$, and only depends on the background cosmology and the formation redshift $z_{f}$. The function $T_{0}(R)$ is the Radius-Temperature relation for collapse at $z_{f}=0$, for a given background cosmology, thus $\phi\left(z_{f}=0\right)=1$. Equation (18) was derived previously by Eke et al. (1996). The factor $\Delta_{c} \equiv 3 M /\left(4 \pi r_{\text {vir }}^{3} \rho_{c}\right)$ is the ratio of the virialized physical mass density to the critical (not the mean) cosmological density at the time of collapse, and $r_{\text {vir }}$ is taken to be $r_{\max } / 2$. In an $\Omega=1$, matter dominated universe, $\Delta_{c}=18 \pi^{2} \approx 178$; for $\Omega<1, \Delta_{c}$ can be derived from uniform spherical collapse (see Eke et al. 1996). The quantity $\Delta_{c}$ depends only on the background cosmology. Because $\Delta_{c}$ and $\Omega$ asymptote to $18 \pi^{2}$ and 1 respectively as $z_{f} \rightarrow \infty$, and hence change by a factor $<\Omega_{0}^{-1}$, the $z_{f}$-dependence of $\phi$ is dominated by the factor $1+z_{f}$. 
Evrard et al. (1996) have compared numerical simulations for Gaussian $\Omega_{0}=1.0$ and $\Omega_{0}=0.2$ models, and found that $T$ scales as $M^{2 / 3}$, as equation (18) implies. Fitting a power law to their results, and converting mass to comoving scale implies $k T=(8.8 \pm$ $0.8 \mathrm{keV})\left(\Delta_{c} / 18 \pi^{2}\right)^{0.164} \Omega_{0}^{2 / 3}\left(R / 8 h^{-1} \mathrm{Mpc}\right)^{2}$. This equation is consistent with equation (19) for all three background cosmologies we consider, using the results of the next section to determine $z_{f}$ and $\phi\left(z_{f}\right)$. The detailed simulations necessary to check the cluster temperatures for the texture scenario have not been done. Bartlett (1997) notes, however, that for any given density profile of a spherical collapse, the power law $T \propto M^{2 / 3} \propto R^{2}$ should be the same - the only difference might be in the constant of proportionality (e.g. through a different $\phi$-dependence).

Taking the derivative of equation (20) with respect to $R$ gives

$$
\frac{d T}{d R}=T_{0}(R) \frac{d \phi}{d R}+\phi\left[z_{f}\right] \frac{d T_{0}}{d R}=\frac{T}{R}\left(2+\frac{d \ln \phi}{d \ln R}\right),
$$

where the redshift of formation $z_{f}$ must be calculated as a function of $R$. Finally, inverting equation (20) gives $R$ as a function of the temperature:

$$
\begin{aligned}
R & =10 h^{-1} \operatorname{Mpc} \Omega_{0}^{-1 / 3}\left(\frac{T}{8.6 \mathrm{keV}}\right)^{1 / 2}\left(\frac{18 \pi^{2}}{\Delta_{c}(0)}\right)^{1 / 6} \times\left[\left(\frac{\Delta_{c}(0)}{\Delta_{c}\left(z_{f}\right)} \cdot \frac{\Omega\left(z_{f}\right)}{\Omega_{0}}\right)^{1 / 6} \frac{1}{\left(1+z_{f}\right)^{1 / 2}}(22)\right. \\
& \equiv R_{0}(T) \times \phi\left[z_{f}(y)\right]^{-1 / 2}
\end{aligned}
$$

where $\phi$ is defined as above, the factor in front of the square brackets has been replaced by $R_{0}(T)$, the Radius-Temperature relation at $z_{f}=0$. Note that equation (23) so defined is only valid when $z_{f}$ is known.

\subsection{Formation Epoch of Clusters in PS}

We now have another parameter to consider, the redshift of formation $z_{f}$. The standard assumption is to assume that the cluster has "just formed" at the redshift $z_{0}$ at which we observe it, i.e., that $z_{f}=z_{0}$. In the $\Omega_{0}=1$ Gaussian CDM scenario, this assumption is valid, since clusters form late and accretion continues to the present (e.g., Lacey \& Cole 1993). If $\Omega_{0}<1$, however, linear growth of perturbations freezes out when the universe enters its phase of free expansion at $1+z \sim 1 / \Omega_{0}$ (see Peebles 1993). Therefore, a given structure must collapse at a higher redshift than in the $\Omega_{0}=1$ case in order to form. If there is a sufficiently positively-skewed non-Gaussian tail to the distribution function, as there is for textures, a larger fraction of the universe collapses non-linearly early on (see Bartlett et al. 1993 and 
Pen et al. 1994). Furthermore, for textures and other "causal-seed" models, whose density perturbations are compensated, there is a limit to the final mass of a collapsing structure, defined by the size of the causal horizon at the time the perturbation is seeded. Because the integrated overdensity is zero on larger scales, no further accretion can occur (Bartlett et al. 1993).

We now derive the mean redshift of formation $\left\langle z_{f}\right\rangle$, and use that redshift in equation (20) to determine the typical temperature of clusters as a function of $R$. The net differential number density of clusters which form in redshift interval $(z, z+d z)$ follows from equation (13):

$$
\frac{d n_{R}}{d z} d z=\frac{3}{4 \pi R^{3}} \frac{\alpha}{R} f \frac{d}{d z}\{y(z) P[y(z)]\} d z
$$

where

$$
\begin{aligned}
y(z) \equiv \frac{\delta_{c}(z)}{\sigma_{R}(z)} & =\frac{\delta_{c}(0)}{\sigma_{R}(0)} \cdot \frac{\delta_{c}(z)}{\delta_{c}(0) D(z)} \\
& \equiv y_{0} \frac{\delta_{c}(z)}{\delta_{c}(0) D(z)} .
\end{aligned}
$$

Here $y_{0}$ refers to the value of $y(z)$ at $z=0, D(z)$ is the linear growth factor normalized to unity at redshift zero, and $\delta_{c}(z)$ is the critical linear density of collapse at redshift $z$. For clusters observed at redshift $z_{0}$, the mean redshift of formation $\left\langle z_{f}\right\rangle_{z_{0}}$ is obtained by averaging the redshift $z$ weighted by equation (24):

$$
\begin{aligned}
\left\langle z_{f}\right\rangle_{z_{0}} & =\frac{\int_{z_{0}}^{\infty} z \frac{d n_{R}}{d z} d z}{\int_{z_{0}}^{\infty} \frac{d n_{R}}{d z} d z} \\
& =z_{0}+\frac{1}{y\left(z_{0}\right) P\left[y\left(z_{0}\right)\right]} \int_{z_{0}}^{\infty} y(z) P[y(z)] d z
\end{aligned}
$$

where the second line comes from integrating the numerator by parts, integrating the denominator, and cancelling terms. The mean redshift of formation of clusters at $z_{0}$ is a function only of the background cosmology through $\delta_{c}(z)$ and $D(z)$, and the current value of $y_{0}$ through equation (26). We take the formation redshift required in equation (20) to be $\left\langle z_{f}\right\rangle$. When using equation (28) with the present epoch abundances below, we set $z_{0}=0$.

\subsection{Determining $\eta$ from X-ray Cluster Surveys}

Using equations (20) and (21) in equation (14) gives

$$
2 n_{T} T\left(1+\frac{\alpha}{2} \frac{d \ln \phi}{d \ln y}\right)=\frac{3}{4 \pi R^{3}} \alpha y f P(y)
$$


where $y$ is now understood to be its present day value, and we have used $\frac{d \ln y}{d \ln R}=-\frac{d \ln \eta}{d \ln R}=\alpha$, because of our power-law assumption. Replacing $R$ with equation (23) and separating those quantities depending on $T$ from those depending on $y$ gives

$$
n_{T} T \frac{8 \pi R_{0}^{3}(T)}{3}=\frac{\phi^{\frac{3}{2}}}{1+\frac{\alpha}{2} \frac{d \ln \phi}{d \ln y}} \alpha y f P(y) .
$$

We need now only to relate observations of clusters to the left-hand-side of equation (30).

There are two virtually complete X-ray flux-limited surveys of rich galaxy clusters at low redshift. The first was compiled by Henry \& Arnaud (1991), and the second is the "X-ray Brightest Abell Cluster Survey" (XBACS) from the ROSAT all-sky survey (Ebeling et al. 1996). The Henry \& Arnaud (1991) survey is essentially contained within the XBACS survey. An unbiased estimator of the number density of clusters in the temperature interval $(T, T+\Delta T)$ for a flux-limited survey is given by

$$
n_{T} \Delta T=\sum_{T<T_{i}<T+\Delta T} V_{\max , i}^{-1}
$$

where $V_{\max , i}$ is the maximum volume in which the cluster of temperature $T_{i}$ (in keV) could be detected given the flux limit and geometric boundaries of the survey. In this case, we require a geometrical boundary at galactic latitude $|b|>20^{\circ}$, and redshift $z \leq 0.1$. The full XBACS sample takes the maximum redshift to be $z_{\max }=0.2$. The Poisson variance, following Pen (1997), is given by:

$$
\sigma^{2}\left(n_{T} \Delta T\right)=\sum_{T<T_{i}<T+\Delta T} V_{\max , i}^{-2}
$$

The cumulative temperature functions $n(>T)$ for the two samples are shown in Figure 4 . The two temperature functions are quite consistent in the overlapping temperature range $>3 \mathrm{keV}$, so we use this part of the $(10 \times)$ larger XBACS survey for our analysis.

Substituting equation (31) gives, finally,

$$
\left(\frac{T}{\Delta T} \sum_{T<T_{i}<T+\Delta T} V_{\max , i}^{-1}\right) \frac{8 \pi R_{0}^{3}(T)}{3}=\frac{\phi^{\frac{3}{2}} \alpha y f P(y)}{1+\frac{\alpha}{2} \frac{d \ln \phi}{d \ln y}} .
$$

We define the temperature bins $(T, T+\Delta T)$ so that there are at least two clusters in each. For a fixed background cosmology and power law index $\alpha$, the left-hand side of equation (33) contains only observed quantities, and the right-hand side is a function of $y$ and the PDF. For each PDF, we therefore can solve for $y$ implicitly as a function of $T$. We then convert $T$ to $R$ using equation (23) and our derived value of $y$, and obtain a value of $\sigma_{R}^{\text {clusters }} \equiv \delta_{c} / y$ as 
a function of the value of $R$ inferred from each temperature bin. Multiplying by $\Omega_{0}^{0.6}$ gives the cluster-inferred $\eta_{R}^{\text {clusters }}$, which can be compared directly with the values from the rms measurements.

A clear comparison with rms-inferred $\eta$ can be made if we extrapolate all values of $\eta$ to a common scale, using the power law model we have assumed throughout. We choose $10 h^{-1} \mathrm{Mpc}$ because this scale corresponds to a temperature within the range of the observations for $\Omega_{0}=0.3-1.0: 8.6 \mathrm{keV}$ for $\Omega_{0}=1$ and $3.4 \sim 4.0 \mathrm{keV}$ (depending on $\phi$ ) for $\Omega_{0}=0.3$. For $\Omega_{0}=0.3$, the scale $R=8 h^{-1} \mathrm{Mpc}$ corresponds to a temperature of $2.2 \sim 2.6$ $\mathrm{keV}$, outside the range of observations we consider.

For each temperature bin, we therefore extrapolate the derived value of $\eta_{R}$ to $10 h^{-1} \mathrm{Mpc}$ using $\eta_{R} \propto \sigma_{R} \propto R^{-\alpha}$, and obtain a measurement of $\eta_{10}$ for each bin. We wish to find the resulting probability distribution in $\eta_{10}$. We find that the statistical errors in $\eta_{10}$ from equations (32) and (33) are smaller than the scatter of the values of $\eta_{10}$, though the discrepancies are not as drastic as in the case of the rms measurements. Certainly, then, there remains some type of systematic error. We sum the distribution functions of each extrapolated $\eta_{10}$ to obtain the total distribution function, assuming each error is Gaussian:

$$
\mathcal{P}\left(\eta_{10} \mid D_{\text {clus }} \mathrm{PDF}\right)=\frac{1}{N} \sum_{i=1}^{N} \frac{1}{\sqrt{2 \pi \sigma_{i}^{2}}} e^{-\frac{1}{2}\left(\frac{\eta_{10, i}-\eta_{10}}{\sigma_{i}}\right)^{2}},
$$

where the sum is over the temperature bins labeled by $i$. Note that we are summing over the individual distributions because the individual temperature bins are not independent measures of $\eta_{10}$; i.e., the data set as a whole is taken as a single measurement, with the error given by the total distribution $\mathcal{P}\left(\eta_{10} \mid D_{\text {clus }} \mathrm{PDF}\right)$.

Figures 5 5 show the cluster-inferred $\eta_{10}$ distributions for power laws $\alpha=0.83,0.60$, and 1.10, and for different values of $\Omega_{0}$ and the PDF. Also shown are the likelihoods for mean peculiar velocity/redshift distortion-derived rms values discussed in $\S 2$, and the Willick et al. (1997b) result alone. The means and standard deviations of the resulting distribution of $\eta_{10}$ measurements are given in Table 2, for various values of $\Omega_{0}$ and $\alpha$.

The most prominent feature of the inferred rms from clusters is its strong dependence on the PDF. Gaussian and texture models give clearly different predictions for any given cluster data set. The degree to which the cluster-inferred value of $\eta_{10}$ differs between Gaussian and texture PDFs is directly related to the degree to which the tail of the texture PDF is distinguishable from a Gaussian.

\section{Discussion}




\subsection{Which PDF is More Likely?}

We quantify the comparison between the rms and cluster-inferred determinations of $\eta_{10}$ with a Bayesian model comparison analysis (Loredo 1990). For now, we fix the background cosmology and the power law index $\alpha$, and suppress the dependence on these assumptions. We ultimately seek the probability that a certain PDF is correct, given the cluster data $D_{\text {clus }}$ and the rms data $D_{\text {rms }}$ :

$$
\mathcal{P}\left(\mathrm{PDF} \mid D_{\text {clus }} D_{\text {rms }}\right)=\frac{\mathcal{P}(\mathrm{PDF})}{\mathcal{P}\left(D_{\text {clus }} D_{\text {rms }}\right)} \mathcal{P}\left(D_{\text {clus }} D_{\text {rms }} \mid \mathrm{PDF}\right)
$$

where we have used Bayes Theorem. The fraction in front consists of a prior (which we take as uniform) and a normalization factor. Using the product rule, we separate the last probability:

$$
\mathcal{P}\left(D_{\text {clus }} D_{\text {rms }} \mid \mathrm{PDF}\right)=\mathcal{P}\left(D_{\text {clus }} \mid \mathrm{PDF}\right) \mathcal{P}\left(D_{\text {rms }} \mid D_{\text {clus }} \mathrm{PDF}\right)
$$

Since we are primarily interested in the rms-versus-tail comparison, we take the first probability in equation (36) to be independent of the PDF. A more sophisticated analysis would evaluate the "goodness of fit" of the cluster data for each PDF, and thereby estimate the absolute probability $\mathcal{P}\left(D_{\text {clus }} \mid \mathrm{PDF}\right)$. However, as mentioned in $\S 3$, the scatter in the inferred values of $\eta_{10 \text {,clus }}$ is greater than the calculated Poisson errors, so any such analysis will have to take into account unknown systematic errors (e.g. using the model described in $\S 2.2$ ).

The dependence of $D_{\text {rms }}$ on $D_{\text {clus }}$ in the second probability in equation (36) is through the value of $\eta_{10}$; using the summation rule gives

$$
\mathcal{P}\left(D_{\text {rms }} \mid D_{\text {clus }} \mathrm{PDF}\right)=\int d \eta_{10} \mathcal{P}\left(D_{\text {rms }} \mid \eta_{10} \mathrm{PDF}\right) \mathcal{P}\left(\eta_{10} \mid D_{\text {clus }} \mathrm{PDF}\right)
$$

The resulting expression for equation (35), suppressing all factors assumed to be independent of the PDF, is

$$
\mathcal{P}\left(\mathrm{PDF} \mid D_{\text {clus }} D_{\text {rms }}\right) \propto \int d \eta_{10} \mathcal{P}\left(D_{\text {rms }} \mid \eta_{10} \mathrm{PDF}\right) \mathcal{P}\left(\eta_{10} \mid D_{\text {clus }} \mathrm{PDF}\right)
$$

The cluster distribution function in $\eta_{10}, \mathcal{P}\left(\eta_{10} \mid D_{\text {clus }} \mathrm{PDF}\right)$, is given by equation (34). The rms probability depends only the power law slope $\alpha$, and the weighting of the different analyses, and is independent of the PDF. If we use the error model in $\S 2.2$, the probability density $\mathcal{P}\left(D_{\text {rms }} \mid \eta_{10} \mathrm{PDF}\right)$ is given by the likelihood function from equation (11) and Figure 2,

$$
\mathcal{P}\left(D_{\text {rms }} \mid \eta_{10} \mathrm{PDF}\right)=\mathcal{L}_{\text {rms }}\left(\eta_{10}\right)
$$

If we are considering only the Willick et al. (1997b) data, $D_{\text {Willick, }}$ and we assume normal errors, we use

$$
\mathcal{P}\left(D_{\text {Willick }} \mid \eta_{10} \mathrm{PDF}\right)=\frac{1}{\sqrt{2 \pi \epsilon_{\mathrm{W}}^{2}}} \exp \left[-\frac{1}{2}\left(\frac{\eta_{10}-\eta_{\mathrm{W}}}{\epsilon_{\mathrm{W}}}\right)^{2}\right]
$$


where $\eta_{\mathrm{W}}$ and $\epsilon_{\mathrm{W}}$ are given by the values inferred from their analysis, also listed in Table 1 .

Since we do not know the full range of possible PDFs, we take the Bayesian approach of considering the likelihood ratio (the "odds", or "Bayes factor" for uniform priors, Loredo 1990) of a Gaussian versus a texture PDF. The values of the likelihood ratio $\mathcal{P}$ (Gaussian) $/ \mathcal{P}$ (texture) are listed in Table 3, for various background cosmologies and values of $\alpha$. If we take the average of the rms measurements, a Gaussian PDF is favored for any background cosmology. The probability of a Gaussian PDF is always $>2 \times$ that of a texture PDF. The confidence level of the accepting a Gaussian hypothesis is $70-88 \%$, roughly $1 \sigma \sim 1.5 \sigma$. If we only use the Willick et al. (1997b) measurement, however, then which PDF is favored depends strongly on the background cosmology, and somewhat on the power law index $\alpha$. In the case of $\alpha=0.83$, if we live in an open $\Omega_{0}=0.3$ universe, then the Gaussian PDF is $3 \times$ more likely than is the texture PDF; if we have a flat $\Omega_{0}=0.3$ universe $(\Lambda=0.7)$, then a Gaussian and texture PDF are roughly equally likely; and if $\Omega_{0}=1$, a texture PDF is $3 \times$ more likely than a Gaussian.

\subsection{Constraints from Cluster Evolution?}

We have only considered the present day abundances of clusters, and we have a degeneracy between the assumed PDF and the background cosmology. The evolution of cluster abundances, however, offers an opportunity to break this degeneracy. In particular, high $\Omega_{0}$ universes evolve more rapidly than those with low $\Omega_{0}$. Gaussian models with $\Omega_{0}=1$ are already heavily disfavored because they predict too rapid an evolution in cluster abundances out to $z \geq 0.3$ (Henry 1997; Donahue et al. 1997). The fact that the Willick et al. (1997b) measurement leads to the $\Omega_{0}=1$ Gaussian scenario being disfavored relative to a texture scenario simply reinforces the case against a flat matter-dominated universe for Gaussian fluctuations.

The formalism of $\S 3$ relating cluster abundances to the PDF and the value of $\eta$ is fully generalizable to $z>0$. As an example, we show in Figure 8 the cumulative number density of clusters $\geq 10 \mathrm{keV}$, as a function of redshift for various scenarios. These curves are normalized to a present-day number density of $1 \times 10^{-8} h^{3} \mathrm{Mpc}^{-3}$ (see Figure (i). For fixed background cosmology, the texture scenarios evolve slower than the Gaussian ones because of the shallower texture PDF. For fixed PDF, the evolution of the number density evolves fastest with $\Omega_{0}=1$ and slowest with open $\Omega_{0}=0.3$. A Gaussian PDF with a flat $\Omega_{0}=0.3$ background cosmology has roughly the same evolutionary history as a texture PDF with $\Omega_{0}=1$. If evolution is observed to be significantly slower or more rapid than this, then an $\Omega_{0}=1$ texture scenario can be ruled out. 
Henry (1997) reports that the number density of $z=0.3$ clusters at the hot end of the temperature function ( $\gtrsim 6.5 \mathrm{keV}$ ) is a factor of $\sim 2.5$ lower than today (his Figure 2), though this is still within the calculated scatter due to Poisson statistics. For $\Omega_{0}=1$, the Gaussian model predicts a factor $\sim 8$ decrease in the density of such clusters at $z=0.3$ while the texture model predicts a factor $\sim 3$ decrease. For the $\Omega_{0}=0.3$ scenarios, the reduction factor is $1.1 \sim 2$. Henry (1997) reports that the cluster evolutionary data rule out the Gaussian $\Omega_{0}=1$ at $99 \%$ confidence.

Donahue et al. (1997) report an extremely hot X-ray temperature of $14.7 \pm 4 \mathrm{keV}$ for cluster MS1054-03, at redshift 0.828. They indicate that this result, along with data from other high-redshift clusters they have studied, implies that, within errors of $\sim 50 \%$, the number density of clusters with $T>10 \mathrm{keV}$ is essentially unchanged from $z=0$ to $z=0.8$. If this result is confirmed, even a texture PDF would be ruled out for $\Omega_{0}=1$, since it predicts a decrease in the number density by a factor of $\sim 30$. Further observations at redshift $z=0.5 \sim 1.0$ will almost certainly resolve the ambiguity between models with different $\Omega_{0}$.

\section{Summary and Conclusions}

We show that comparing the amplitude of tail fluctuations indicated by the abundances of clusters of galaxies, to the amplitude of the rms mass fluctuation as indicated by galaxy peculiar velocities and redshift space distortions, can be a strong test of the Gaussianity of the PDF of the initial density field, with a weak dependence on the slope of the density fluctuation spectrum.

Observations of peculiar velocities and redshift distortions in the linear regime can measure the rms level of gravitational potential fluctuations present in the universe in the combination $\eta_{R} \equiv \sigma_{R} \Omega_{0}^{0.6}$, independent of galaxy bias. Various existing measurements of this quantity are not consistent with one another within their stated errors. We assume the existence of a systematic error in each measurement, and use a maximum likelihood technique to combine these recent measurements of $\eta_{R}$. The systematic error leads to a $\sim 20 \%$ uncertainty in the value of $\eta_{R}$. This procedure gives $\eta_{10, \mathrm{rms}}=0.39 \pm 0.07-0.51 \pm 0.10$, depending on the assumed slope of the mass fluctuation spectrum. We use this value, and

one recent measurement by Willick et al. (1997b), which indicates $\eta_{10}=0.27-0.30 \pm$ 0.04, to compare with the cluster-inferred measures of $\eta_{10}$. We chose the Willick et al. (1997b) measurement because it is the most recent analysis which explicitly filters close to the $10 h^{-1} \mathrm{Mpc}$ scale on which clusters form. 
The value of $\eta_{10}$ inferred from cluster abundances is calculated using the Press-Schechter approach for both a Gaussian and a texture PDF. We find a simple relation for the typical redshift of formation of clusters in both models. This is particularly important in the texture scenario, where the shallower tail of the PDF leads to significant early formation of clusters. This typical redshift is important in the relation between the virial temperature of the clusters and the comoving radius of the initial perturbation. For a texture PDF, the observed abundances of X-ray clusters implies $\eta_{10}=0.20 \pm 0.03-0.25 \pm 0.04$ for $\Omega_{0}=0.3$, and $\eta_{10}=0.22-0.29 \pm 0.03$ for $\Omega_{0}=1.0$. Given a Gaussian PDF, on the other hand, the cluster abundances indicate $\eta_{10}=0.29 \pm 0.04-0.33 \pm 0.05$ for $\Omega_{0}=0.3$ and $\eta_{10}=0.34 \pm 0.05-$ $0.42 \pm 0.04$ for $\Omega_{0}=1$.

We calculate the relative likelihoods of each PDF for a range of cosmological parameters. Using the maximum likelihood average of the rms measurements with the cluster abundances implies the Gaussian model is favored for any $\Omega_{0}$. However, using the Willick et al. (1997b) rms measurement with the clusters implies that a Gaussian is favored for open $\Omega_{0}=0.3$, a Gaussian and a texture PDF of roughly equal likelihood for flat $\Omega_{0}=0.3$, and a texture PDF is favored for $\Omega_{0}=1$. In each case, the accepted hypothesis is accepted at a $\sim 1.5 \sigma$ level, with likelihood ratio $\gtrsim 3$. Preliminary constraints from cluster evolution indicate that low $\Omega_{0}$ models are probably more viable than $\Omega_{0}=1$ models. Our conclusions can are summarized in Table 4 , where a $\sqrt{ }(\mathrm{X})$ indicates the PDF is favored (disfavored) over the alternative by $\gtrsim 3 \times$. A ? next to the $\sqrt{ }$ or $\mathrm{X}$ indicates the factor is $\lesssim 3$.

Our results imply that the textures+CDM scenario faces some challenges from observations on $5-25 h^{-1} \mathrm{Mpc}$ scales, completely independent of CMB measurements. Although the current state of the measurements of the rms prevents us from firmly distinguishing the Gaussian and texture models, the principle behind this method is clear. The observed cluster abundance at $z=0$ predicts significantly different values for $\eta_{10}$ for the two models. More accurate direct determinations of the measured rms from future redshift surveys and peculiar velocity measurements, or from an improved understanding of the systematic effects which are present in the current analyses, will surely enable us to definitively test any distribution function of initial density perturbations. Furthermore, upcoming high redshift X-ray cluster data will likely break any remaining degeneracy due to the assumed background cosmology. Soon, perhaps, we will finally be able to distinguish different models for the initial PDF of the universe at $10 h^{-1} \mathrm{Mpc}$.

We thank Avishai Dekel and Ed Turner for useful conversations. MAS acknowledges the support of the Alfred P. Sloan Foundation, Research Corporation, and NSF Grant AST9616901. 


\section{A. What is the Correct Correspondence Between Tophat and Gaussian Filtering?}

One of the traditional ways of relating different filtering schemes is by matching the volume over which they filter. For tophat and Gaussian, then, this would correspond to $4 \pi R_{T}^{3} / 3=\left(2 \pi R_{G}^{2}\right)^{3 / 2}$, or $R_{T} \approx R_{G} / 1.6$. While this might be appropriate when relating the mass of objects picked out by these filter scales, it is not the best way of relating the wavelengths of fluctuations measured by each filter. Here we derive a method of relating tophat- and Gaussian-filtered fields by their maximum correlation.

Consider a homogeneous, isotropic scalar field $F(\mathbf{x})$ and its Fourier transform in the following convention:

$$
\begin{aligned}
F(\mathbf{x}) & =\sum_{\mathbf{k}} \widetilde{F}(\mathbf{k}) e^{i \mathbf{k} \cdot \mathbf{x}}=\frac{V}{(2 \pi)^{3}} \int d^{3} k \widetilde{F}(\mathbf{k}) e^{i \mathbf{k} \cdot \mathbf{x}} \\
\widetilde{F}(\mathbf{k}) & =\frac{1}{V} \int_{V} d^{3} x F(\mathbf{x}) e^{-i \mathbf{k} \cdot \mathbf{x}} .
\end{aligned}
$$

Let the power spectrum $P(k) \equiv\left\langle|\widetilde{F}(\mathbf{k})|^{2}\right\rangle$. The (dimensionless) tophat and Gaussian filters and their Fourier transforms are then

$$
\begin{aligned}
W_{R_{T}}(\mathbf{x}) & =V \frac{3}{4 \pi R_{T}^{3}} \quad \text { if }|\mathbf{x}|<R_{T}, \quad 0 \text { otherwise } \\
\widetilde{W}_{R_{T}}(\mathbf{k}) & =\frac{3 j_{1}\left(k R_{T}\right)}{k R_{T}} \equiv \widetilde{W}_{T}\left(k R_{T}\right) \\
W_{R_{G}}(\mathbf{x}) & =V \frac{1}{\left(2 \pi R_{G}^{2}\right)^{3 / 2}} \exp \left(-x^{2} /\left(2 R_{G}^{2}\right)\right) \\
\widetilde{W}_{R_{G}}(\mathbf{k}) & =\exp \left(-k^{2} R_{G}^{2} / 2\right) \equiv \widetilde{W}_{G}\left(k R_{G}\right)
\end{aligned}
$$

where $j_{1}(x)$ is the spherical Bessel function of order 1. The filtered fields are $F_{R_{T, G}}(\mathbf{x})=$ $V^{-1} \int d^{3} y F(\mathbf{x}-\mathbf{y}) W_{R_{T, G}}(\mathbf{y})$. Using the convolution theorem, the variance for tophat and Gaussian filters is:

$$
\sigma_{T, G}^{2}\left(R_{T, G}\right)=\frac{V}{(2 \pi)^{3}} \int d k 4 \pi k^{2} P(k) \widetilde{W}_{T, G}^{2}\left(k R_{T, G}\right) .
$$

How do we relate results with a tophat filter and those with a Gaussian filter? We maximize the correlation between the two filtered fields, normalized by their respective standard deviations:

$$
\rho\left(R_{T}, R_{G}\right) \equiv\left\langle\frac{F_{R_{T}}(\mathbf{x})}{\sigma_{T}\left(R_{T}\right)} \frac{F_{R_{G}}(\mathbf{x})}{\sigma_{G}\left(R_{G}\right)}\right\rangle=\frac{1}{\sigma_{T}\left(R_{T}\right) \sigma_{G}\left(R_{G}\right)} V \int \frac{d k 4 \pi k^{2}}{(2 \pi)^{3}} P(k) \widetilde{W}_{T}\left(k R_{T}\right) \widetilde{W}_{G}\left(k R_{G}\right) .
$$


For Gaussian Random Fields, the one-point distribution of each of the filtered fields is Gaussian, and the joint distribution of the two fields is a bivariate Gaussian. In this case the quantity $\rho\left(R_{T}, R_{G}\right)$ is precisely the normalized correlation coefficient of the joint distribution of the two fields. The two filtering schemes, therefore, are most correlated when $\rho\left(R_{T}, R_{G}\right)$ is maximized. In Table A1, we list the peak correlations for power-law power spectra $P(k) \propto k^{n}$ for $n=-2,-1,0$, and 1. For decreasing power spectra $(n<0)$, the correlation between tophat and Gaussian filtering is very good, greater than $87 \%$, with the ratio $R_{T} / R_{G}=1.75 \sim$ 2.

It should be noted that we are not comparing the value of $\sigma$ between the two filtering schemes - we are seeking the scales at which the two filters are most correlated. For example, for a CDM, $\Omega_{0}=1, n=1$, power spectrum, using the maximum correlation, $12 h^{-1} \mathrm{Mpc}$ Gaussian smoothing corresponds to $22.7 h^{-1} \mathrm{Mpc}$ tophat smoothing; but $\sigma_{G}(12) / \sigma_{T}(22.7)=$ 0.86 , while $\sigma_{G}(12) / \sigma_{T}(25.4)=1.0$. 


\section{REFERENCES}

Bahcall, N. 1996, preprint, astro-ph/9611148

Bahcall, N., \& Cen, R. 1992, ApJ, 398, 81

Bahcall, N., Fan, X., \& Cen, R. 1997, ApJ, 485, L53

Bartlett, J.G. 1997, preprint, astro-ph/9703090

Bartlett, J.G., Gooding, A.K., \& Spergel, D.N. 1993, ApJ, 403, 1

Bernardeau 1992, ApJ, 392, 1

Bernardeau 1994, A\&A, 291, 697

Bertschinger, E., Dekel, A., Faber, S.M., Dressler, A., \& Burstein, D. 1990, ApJ, 364, 370

Burstein, D. 1989, privately circulated computer files

Cen, R., et al. 1997, in preparation

Cole, S., Fisher, K.B., \& Weinberg, D.H. 1994, MNRAS, 267, 785

Cole, S., Fisher, K.B., \& Weinberg, D.H. 1995, MNRAS, 275, 515

Davis, M. \& Peebles, P.J.E. 1983, ApJ, 372, 394

Dekel, A., Bertschinger, E., \& Faber, S. M. 1990, ApJ, 364, 349

Donahue, M., Gioia, I., Luppino, G., Hughes, J.P., \& Stocke, J.T., preprint, astroph/9707010

Ebeling, H., Voges, W., Bohringer, H., Edge, A.C., Huchra, J.P, \& Briel, U.G., MNRAS, 281,799

Eke, V.R., Cole, S., \& Frenk, C.S. 1996, MNRAS, 282, 263

Evrard, A.E., Metzler, C.A., \& Navarro, J.F. 1996, ApJ, 469, 494

Fan, X., Bahcall, N., \& Cen, R. 1997, submitted to ApJ

Fisher, K.B., Davis, M., Strauss, M.A., Yahil, A., \& Huchra, J.P. 1994a, MNRAS, 266, 50

Fisher, K.B., Davis, M., Strauss, M.A., Yahil, A., \& Huchra, J.P. 1994b, MNRAS, 267, 927

Fisher, K.B., Scharf, C.A., \& Lahav, O. 1994, MNRAS, 266, 219 
Fry, J. N. 1984a, ApJ, 277, L5

Fry, J. N. 1984b, ApJ, 279, 499

Gooding, A.K., Park, C., Spergel, D.N., Turok, N., \& Gott III, J.R. 1992, ApJ, 393, 42

Gooding, A.K., Spergel, D.N., \& Turok, N. 1991, ApJ, 372, L5

Heavens, A.F. \& Taylor, A.N. 1995, MNRAS, 275, 483

Henry, J.P. 1997, ApJLetters, accepted.

Henry, J.P. \& Arnaud, K.A., 1991, ApJ, 372, 637

Kaiser, N. 1987, MNRAS, 227, 1

Kim, R. \& Strauss, M.A., 1997, submitted to ApJ, astro-ph/9702144

Kolatt, T. \& Dekel, A. 1997, ApJ, 479, 592

Lacey, C.G., \& Cole, S. 1993, MNRAS, 262, 627

Loredo, T.J., "From Laplace to Supernova SN 1987A: Bayesian Inference in Astrophysics," in Maximum Entropy and Bayesian Methods, ed. P.F Fougère, (Dordrecht: Kluwer Academic Publishers), 81-142

Park, C., Spergel, D.N., \& Turok, N. 1991, ApJ, 372, L53

Peebles, P.J.E. 1993, Principles of Physical Cosmology, (Princeton: Princeton University Press)

Pen, U. 1997, preprint, astro-ph/9610147

Pen, U., Seljak, U., \& Turok, N. 1997, preprint, astro-ph/9704165

Pen, U., Spergel, D.N., \& Turok, N. 1994, Phys. Rev. D, 49, 692

Pen, U. \& Spergel, D.N. 1995, Phys. Rev. D, 51, 4099

Press, W.H. \& Schechter, P. 1974, ApJ, 187, 425

Seljak, U., \& Bertschinger, E. 1994, ApJ, 427, 523

Sigad, Y., Eldar, A., Dekel, A., Strauss, M.A., \& Yahil, A. 1997, ApJ, submitted (astroph/9708141) 
Strauss, M.A., \& Willick, J.A. 1995, Physics Reports, 261, 271

Walker, T.P., Steigman, G., Schramm, D.N., Olive, K.A., \& Kang, H.-S. 1991, ApJ, 376, 51

White, S.D.M., Efstathiou, G., \& Frenk, C.S. 1993, MNRAS, 262, 1023

Willick, J.A., Courteau, S., Faber, S.M., Burstein, D., Dekel, A. 1995, ApJ, 446, 12

Willick, J.A., Courteau, S., Faber, S.M., Burstein, D., Dekel, A., \& Kolatt, T. 1996, ApJ, 457,460

Willick, J.A., Courteau, S., Faber, S.M., Burstein, D., Dekel, A., \& Strauss, M.A. 1997a, ApJS, 109, 333

Willick, J.A., Strauss, M.A., Dekel, A., \& Kolatt, T. 1997b, ApJ, 486, 629

Zaroubi, S., Zehavi, I., Dekel, A., Hoffman, Y., \& Kolatt, T. 1997, ApJ, 486, 21 


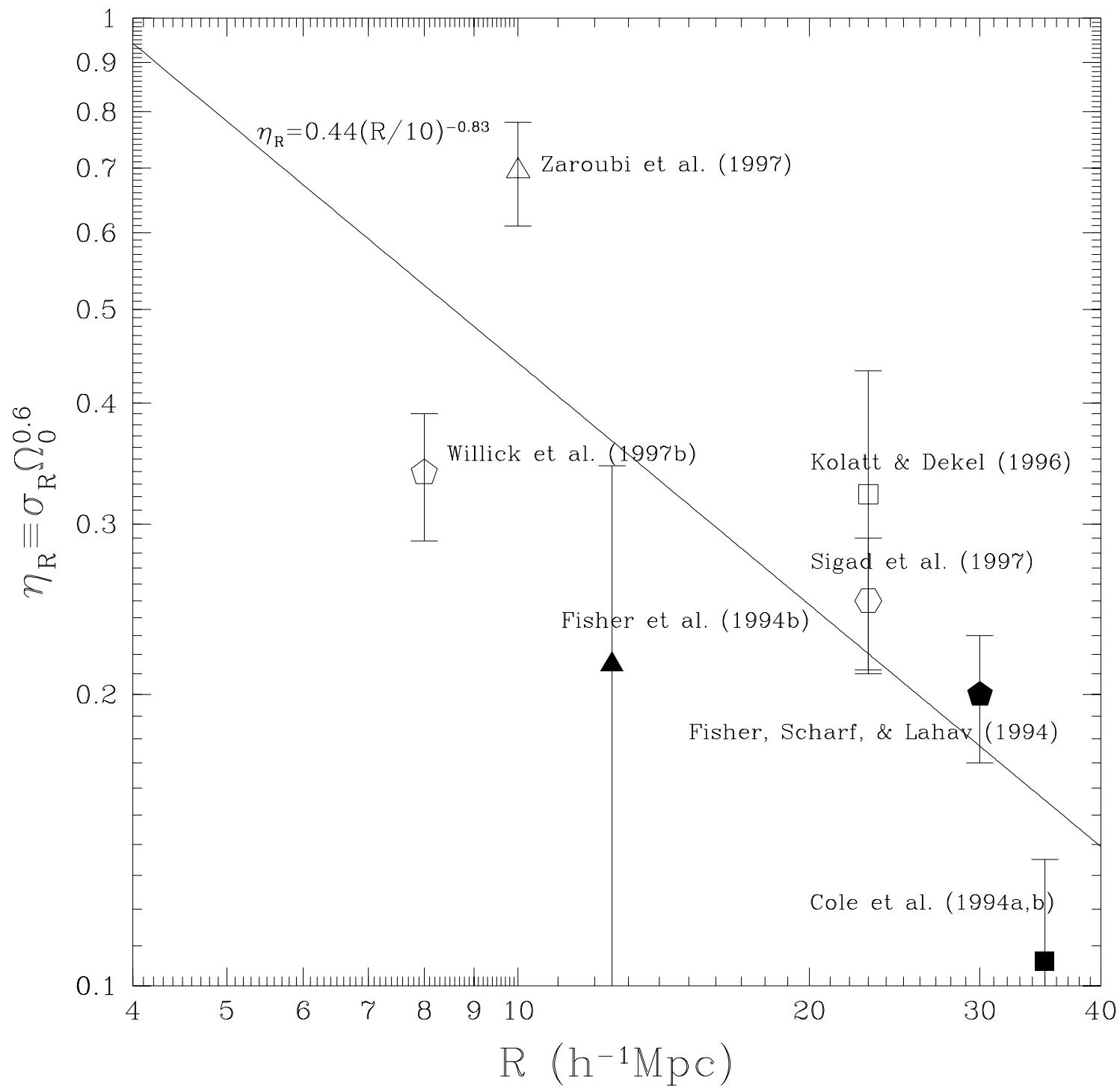

Fig. 1.- RMS mass fluctuations as inferred from peculiar velocity and redshift distortion analyses, as described in $\S 2$, and listed in Table 1. The solid line is $\eta_{R}=$ $0.44\left(R / 10.0 h^{-1} \mathrm{Mpc}\right)^{-0.83}$, where $\alpha$ is that for IRAS galaxies, and $\eta_{10}$ is taken from the maximum likelihood average in Table 1 . 


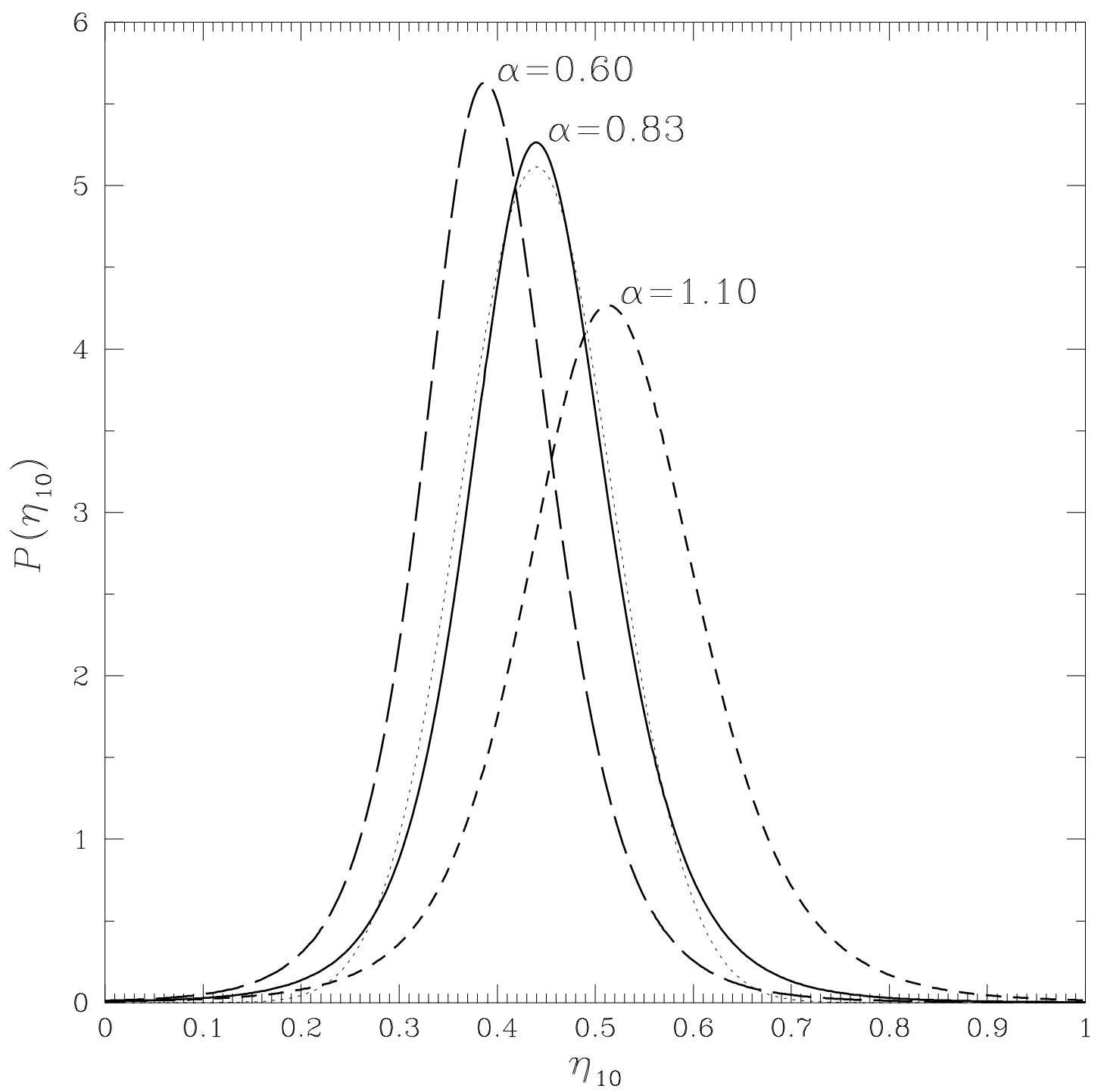

Fig. 2.- Probability density for the error analysis in $\S 2$ of the rms measurements of $\eta_{10}$, for power law indices $\alpha=0.60,0.83$, and 1.10. The thin dotted line is a Gaussian distribution with the same mean and $68 \%$ confidence interval ( $1 \sigma$ error) as the probability density for $\alpha=0.83$. 


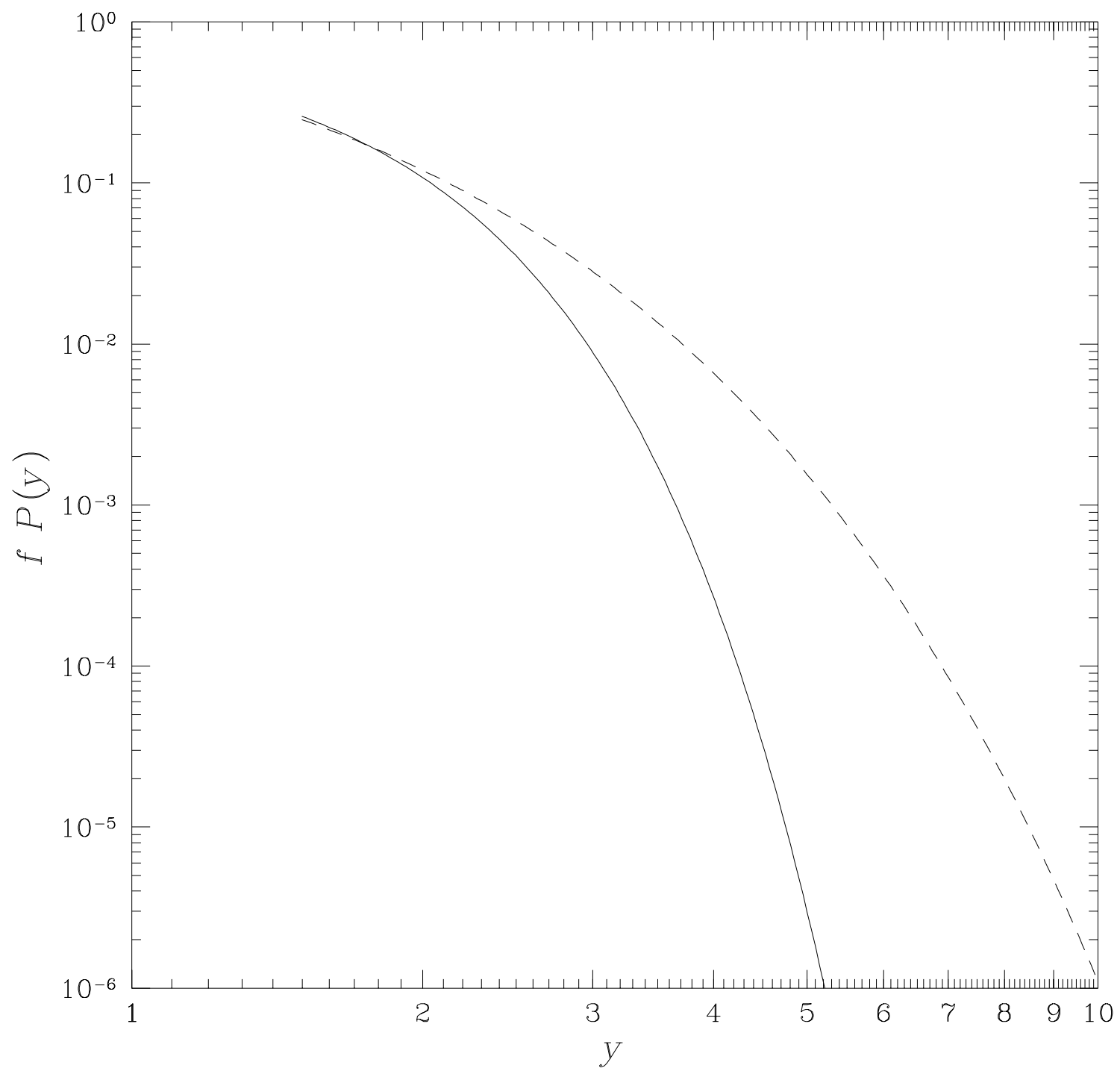

Fig. 3. - Tails of the effective distribution functions $f P(y)$, for a Gaussian (solid) and texture (dashed) PDF. 


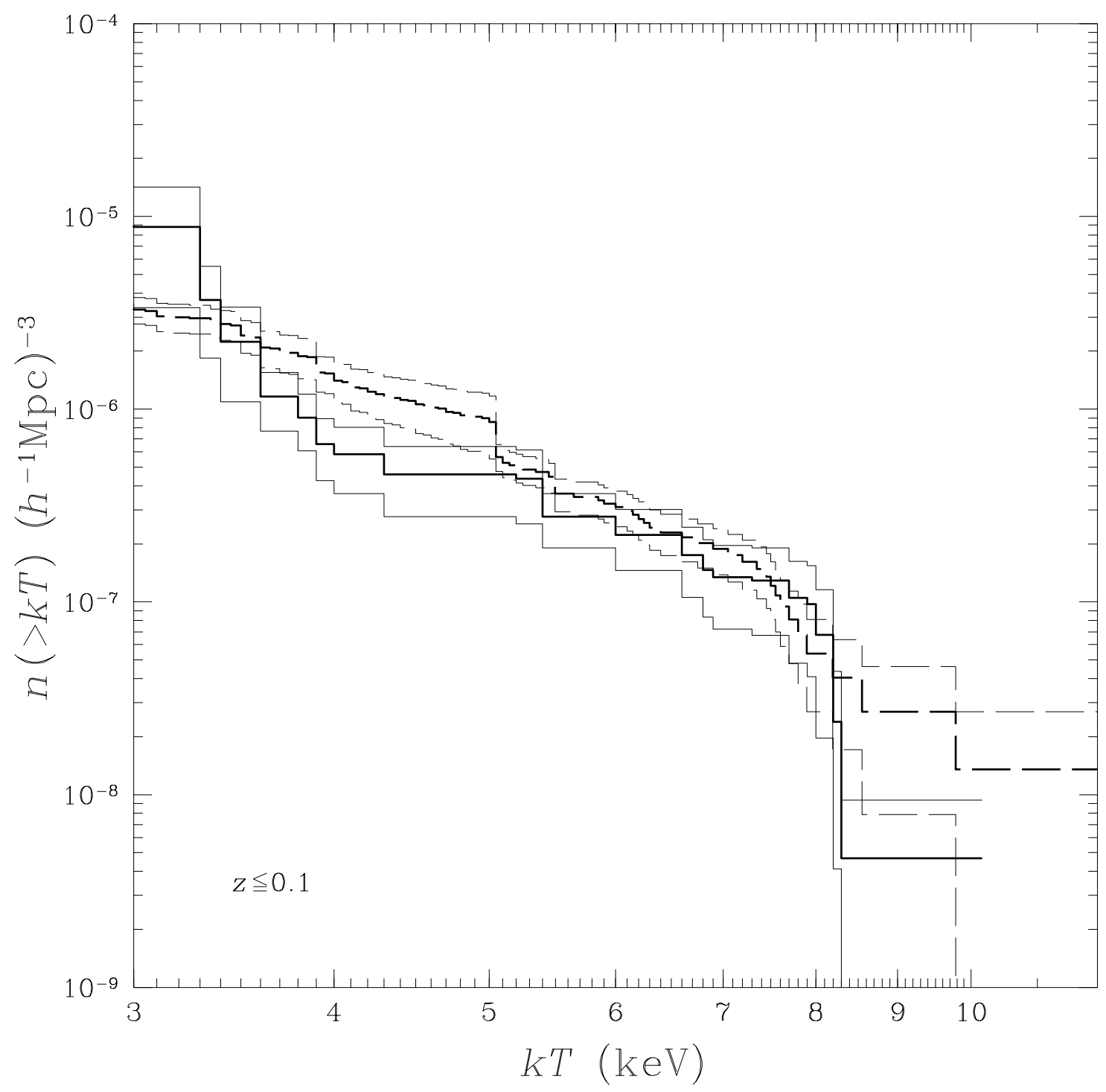

Fig. 4.- Cumulative temperature function from Henry \& Arnaud (1991) (thick solid line), and from the XBACS survey (Ebeling et al. 1996) (thick dashed line), both with $1 \sigma$ Poisson errors from equation (32) (thin histograms). 


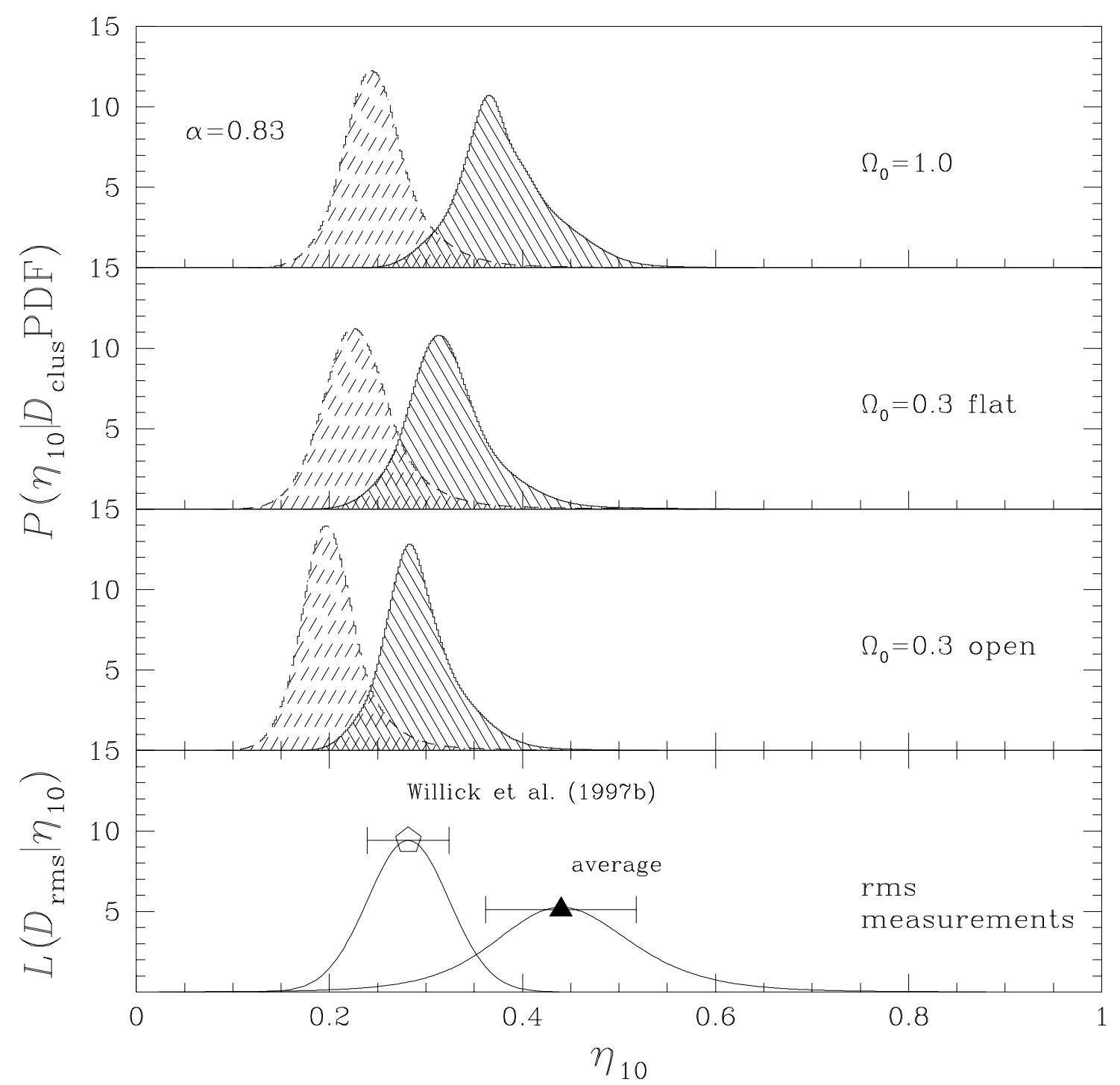

Fig. 5.- Cluster-inferred $\eta_{10}$ compared with peculiar velocity/redshift distortion-inferred $\eta_{10}$ at $10 h^{-1} \mathrm{Mpc}$ for $\alpha=0.83$, for various assumed background cosmologies. The solid-filled distribution functions are for a Gaussian PDF, and the dashed-filled distribution functions are for a texture PDF. The filled triangle is the maximum likelihood average of the measurements in Table 1. The open pentagon is the Willick et al. (1997b) result. 


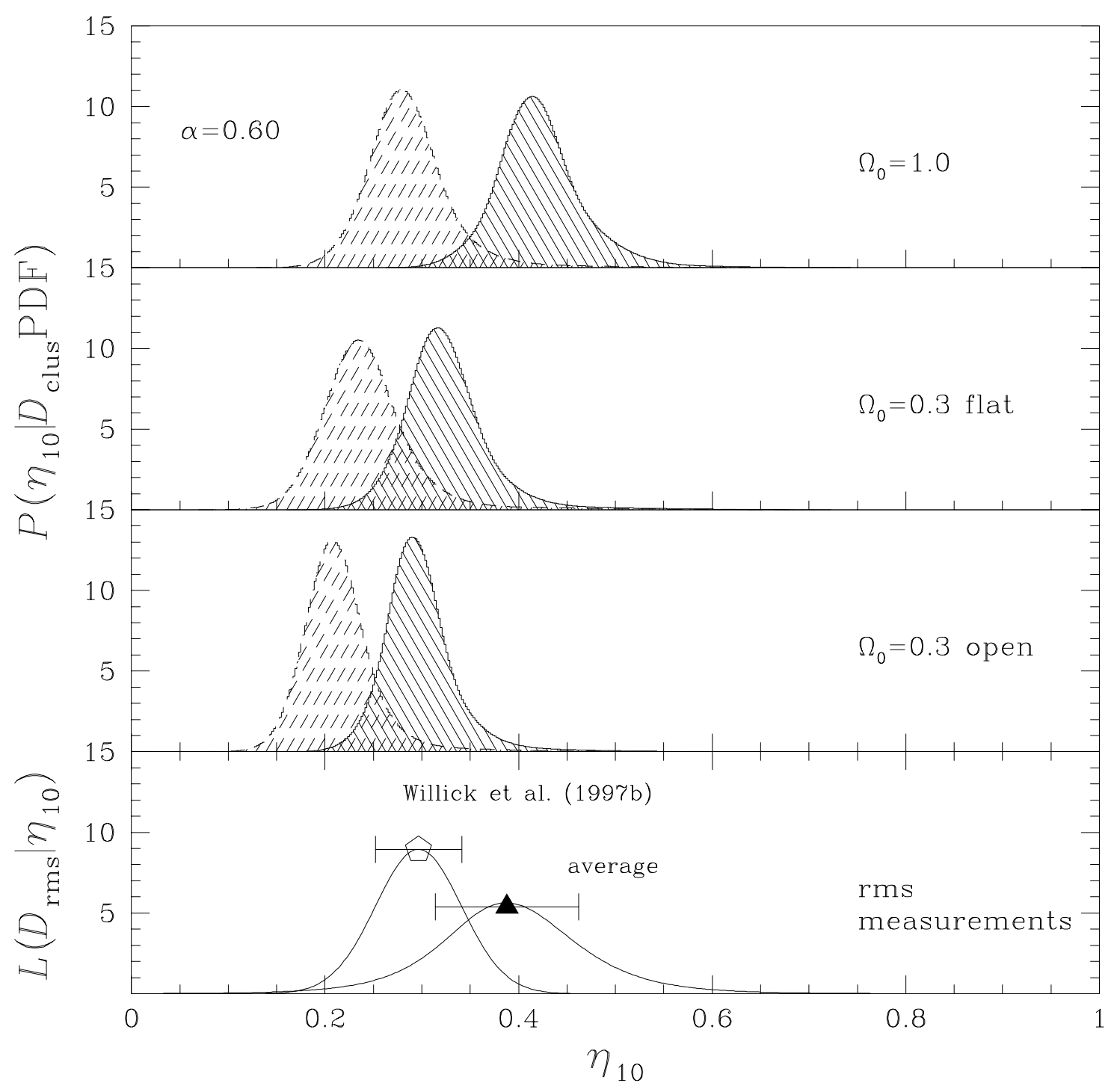

Fig. 6.- Cluster-inferred $\eta_{10}$ compared with peculiar velocity/redshift distortion-inferred $\eta_{10}$ at $10 h^{-1} \mathrm{Mpc}$ for $\alpha=0.60$. The solid-filled distribution functions are for a Gaussian $\mathrm{PDF}$, and the dashed-filled distribution functions are for a texture PDF. 


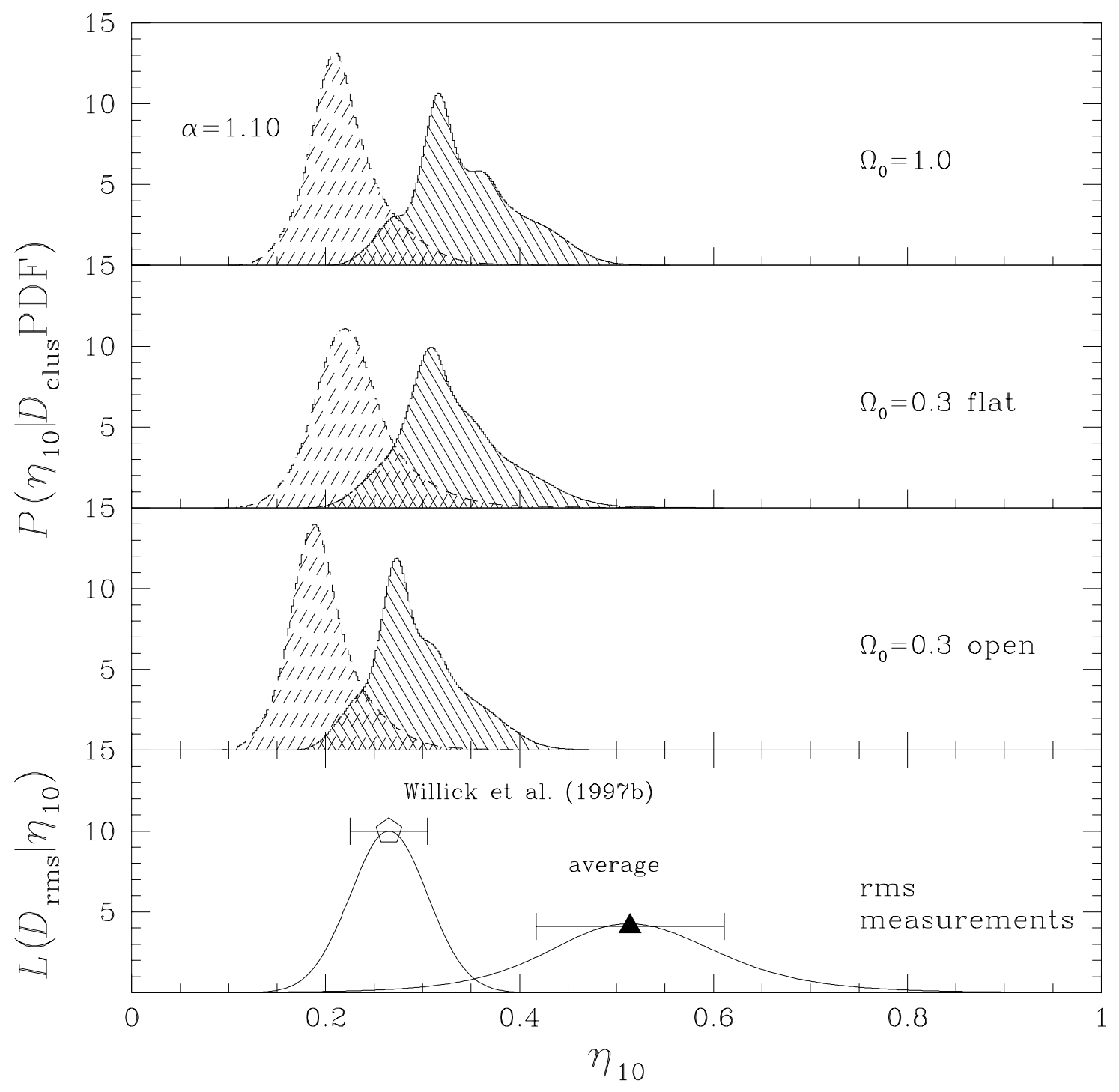

Fig. 7.- Cluster-inferred $\eta_{10}$ compared with peculiar velocity/redshift distortion-inferred $\eta_{10}$ at $10 h^{-1} \mathrm{Mpc}$ for $\alpha=1.10$. The solid-filled distribution functions are for a Gaussian $\mathrm{PDF}$, and the dashed-filled distribution functions are for a texture PDF. 


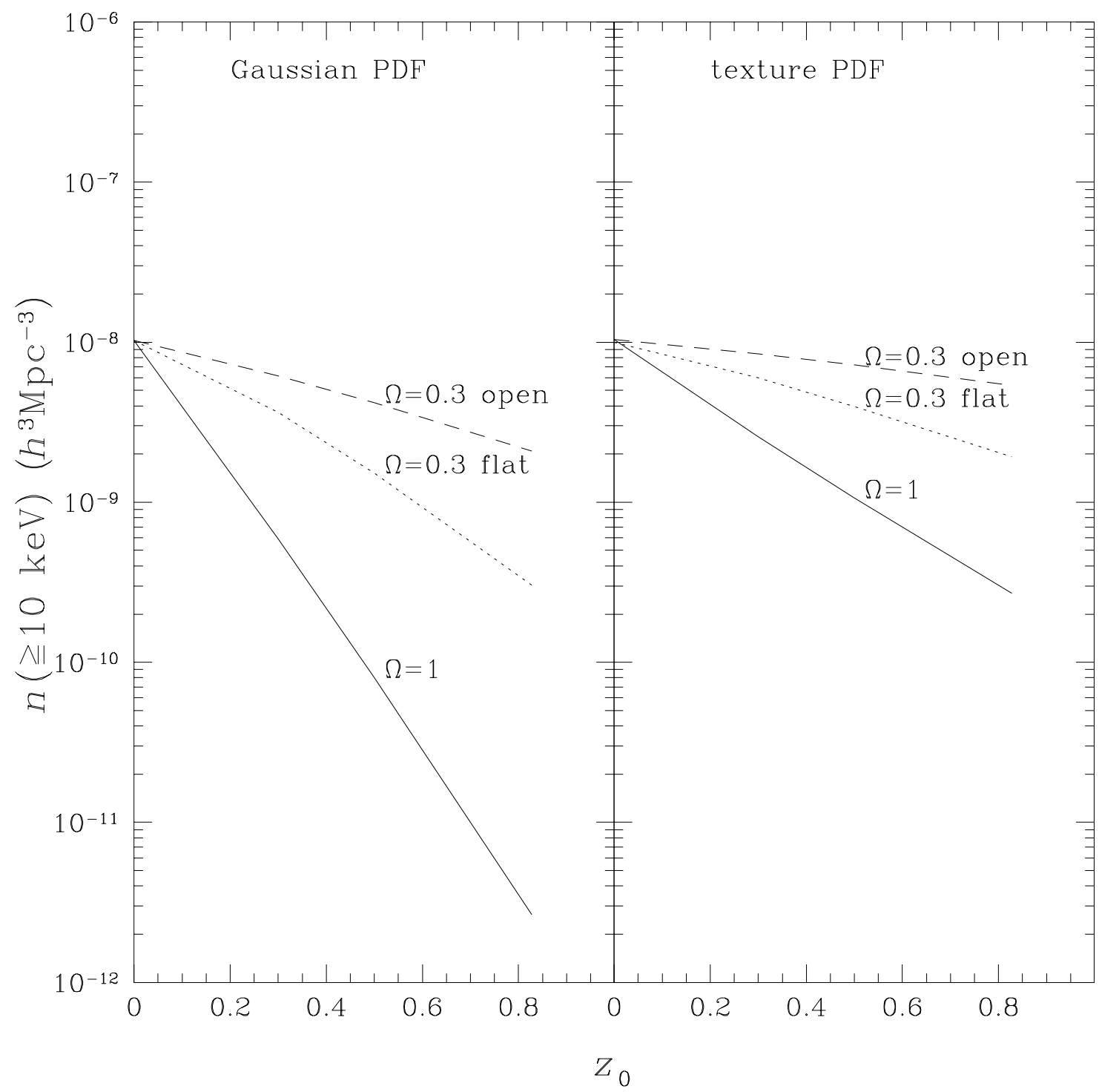

Fig. 8. - Evolution of the number density of clusters with temperature $\geq 10 \mathrm{keV}$, normalized to the present day abundance, for Gaussian and texture PDFs, and various background cosmologies. 
Table 1. RMS Measurements of $\eta_{10}$

\begin{tabular}{lccccc}
\hline \hline \multicolumn{1}{c}{ Reference } & $\mathrm{R}$ & $\eta_{R}$ & & $\eta_{10}$ & \\
& & & $\alpha=0.60$ & $\alpha=\mathbf{0 . 8 3}$ & $\alpha=1.10$ \\
\hline Zaroubi et al. 1997 & 10 & $0.70 \pm 0.09$ & $0.70 \pm 0.09$ & $\mathbf{0 . 7 0} \pm \mathbf{0 . 0 9}$ & $0.70 \pm 0.09$ \\
Kolatt \& Dekel 1996 & 23 & $0.32 \pm 0.11$ & $0.53 \pm 0.18$ & $\mathbf{0 . 6 4} \pm \mathbf{0 . 2 2}$ & $0.80 \pm 0.27$ \\
Willick et al. 1997b & 8 & $0.34 \pm 0.05$ & $0.30 \pm 0.04$ & $\mathbf{0 . 2 8} \pm \mathbf{0 . 0 4}$ & $0.27 \pm 0.04$ \\
\hline Sigad et al. 1997 & 23 & $0.25 \pm 0.04$ & $0.41 \pm 0.07$ & $\mathbf{0 . 5 0} \pm \mathbf{0 . 0 8}$ & $0.62 \pm 0.10$ \\
Fisher et al. 1994b & 12.5 & $0.21 \pm 0.13$ & $0.25 \pm 0.15$ & $\mathbf{0 . 2 6} \pm \mathbf{0 . 1 6}$ & $0.27 \pm 0.17$ \\
Cole et al. 1995a,b & 35 & $0.11 \pm 0.03$ & $0.22 \pm 0.06$ & $\mathbf{0 . 3 0} \pm \mathbf{0 . 0 8}$ & $0.42 \pm 0.12$ \\
Fisher, Scharf, \& Lahav 1994 & 30 & $0.20 \pm 0.03$ & $0.39 \pm 0.06$ & $\mathbf{0 . 5 0} \pm \mathbf{0 . 0 7}$ & $0.67 \pm 0.10$ \\
Maximum Likelihood Avg & 10 & - & $0.39 \pm 0.12$ & $\mathbf{0 . 4 4} \pm \mathbf{0 . 1 2}$ & $0.51 \pm 0.15$ \\
\hline
\end{tabular}

Table 2. XBACS Cluster Measurements of $\eta_{10}$

\begin{tabular}{lccc}
\hline \hline \multicolumn{1}{c}{ Cluster Scenario } & \multicolumn{3}{c}{$\eta_{10}$} \\
& $\alpha=0.60$ & $\alpha=\mathbf{0 . 8 3}$ & $\alpha=1.10$ \\
\hline Gaussian $\Omega_{0}=1$ & $0.423 \pm 0.036$ & $\mathbf{0 . 3 8 1} \pm \mathbf{0 . 0 4 1}$ & $0.342 \pm 0.049$ \\
texture $\Omega_{0}=1$ & $0.287 \pm 0.033$ & $\mathbf{0 . 2 5 2} \pm \mathbf{0 . 0 3 1}$ & $0.220 \pm 0.032$ \\
Gaussian $\Omega_{0}=0.3$ flat & $0.326 \pm 0.035$ & $\mathbf{0 . 3 2 5} \pm \mathbf{0 . 0 3 8}$ & $0.329 \pm 0.047$ \\
texture $\Omega_{0}=0.3$ flat & $0.245 \pm 0.037$ & $\mathbf{0 . 2 3 5} \pm \mathbf{0 . 0 3 4}$ & $0.231 \pm 0.036$ \\
Gaussian $\Omega_{0}=0.3$ open & $0.298 \pm 0.029$ & $\mathbf{0 . 2 9 3} \pm \mathbf{0 . 0 3 3}$ & $0.293 \pm 0.042$ \\
texture $\Omega_{0}=0.3$ open & $0.215 \pm 0.028$ & $\mathbf{0 . 2 0 4} \pm \mathbf{0 . 0 2 7}$ & $0.198 \pm 0.029$ \\
\hline
\end{tabular}


Table 3. Probability of Gaussian (G) Relative to Texture (T) PDF

\begin{tabular}{clccc}
\hline \hline rms & \multicolumn{3}{c}{$\mathcal{P}(\mathrm{G}) / \mathcal{P}(\mathrm{T})$ for $\alpha=$} \\
Averaging & Geometry & 0.60 & $\mathbf{0 . 8 3}$ & 1.10 \\
\hline \multirow{2}{*}{ Maximum } & 1.0 & 2.28 & $\mathbf{6 . 5 2}$ & 7.00 \\
Likelihood & 0.3 flat & 3.28 & $\mathbf{4 . 1 8}$ & 4.41 \\
& 0.3 open & 3.98 & $\mathbf{4 . 7 1}$ & 4.62 \\
Willick et al. & 1.0 & 0.124 & $\mathbf{0 . 3 1 8}$ & 0.704 \\
(1997b) only & 0.3 flat & 1.53 & $\mathbf{1 . 2 5}$ & 0.789 \\
& 0.3 open & 3.28 & $\mathbf{3 . 0 1}$ & 2.13 \\
\hline
\end{tabular}

Table 4. Summary of Tests of Gaussian (G) Versus Textures (T)

\begin{tabular}{|c|c|c|c|c|c|c|}
\hline \multirow[t]{2}{*}{ Tests } & \multicolumn{2}{|c|}{$\Omega_{0}=1$} & \multicolumn{2}{|c|}{$\Omega_{0}=0.3$ flat } & \multicolumn{2}{|c|}{$\Omega_{0}=0.3$ open } \\
\hline & G & $\mathrm{T}$ & G & $\mathrm{T}$ & G & $\mathrm{T}$ \\
\hline rms average and $z=0$ clusters & $\sqrt{ }$ & $\mathrm{X}$ & $\sqrt{ }$ & $\mathrm{X}$ & $\sqrt{ }$ & $\mathrm{X}$ \\
\hline Willick et al. (1997b) and $z=0$ clusters & $\mathrm{X}$ & $\sqrt{ }$ & $?=$ & $?=$ & $\sqrt{ }$ & $\mathrm{X}$ \\
\hline cluster evolution & $\mathrm{X}$ & ?X & $? \sqrt{ }$ & $\sqrt{ }$ & $\sqrt{ }$ & $\sqrt{ }$ \\
\hline
\end{tabular}

Note. - A " $\sqrt{ }$ " ("X") indicates the PDF is favored (disfavored) over the alternative by $\gtrsim 3 \times$ or $\gtrsim 1.5 \sigma$; a "?=" indicates roughly equal likelihood; a "?" next to the " $\sqrt{ }$ " ("X") indicates a $\lesssim 3$ or $\lesssim 1.5 \sigma$ result in favor (against). 
Table A1. Correspondence Between Tophat and Gaussian Filtering

\begin{tabular}{ccc}
\hline \hline $\begin{array}{c}\text { index } n \\
\left(P(k) \propto k^{n}\right)\end{array}$ & $R_{T} / R_{G}$ & $\rho\left(R_{T}, R_{G}\right)$ \\
\hline-2 & (maximum correlation) & \\
-1 & 1.94 & 0.99 \\
0 & 1.85 & 0.97 \\
1 & 1.75 & 0.87 \\
\hline
\end{tabular}

